\title{
Exploiting attractive non-covalent interactions for the enantioselective catalysis of reactions involving radical intermediates
}

\author{
Rupert S. J. Proctor, Avene C. Colgan and Robert J. Phipps* \\ Department of Chemistry, University of Cambridge, Lensfield Road, Cambridge, CB2 1EW. \\ *rjp71@cam.ac.uk
}

The past decade has seen unprecedented growth in the development of new chemical methods that proceed by mechanisms involving radical intermediates. This new attention has served to highlight a long-standing challenge in the field of radical chemistry - that of controlling absolute stereochemistry. This Review will examine developments using a strategy that offers enormous potential, in which attractive non-covalent interactions between a chiral catalyst and the substrate are leveraged to exert enantiocontrol. In a simplistic sense, such an approach mimics the modes of activation and control in enzyme catalysis and the realization that this can be achieved in the context of small-molecule catalysts has had sizable impact on the field of asymmetric catalysis in recent years. This strategy is now starting to quickly gather pace as a powerful approach for control of enantioselectivity in radical reactions and we hope that this focused survey of progress so far will inspire future developments in the area.

The fact that Nature's most fundamental building blocks all exhibit chirality underlines the importance that chemists be able to access synthetic chemical compounds as single enantiomers. The branch of synthetic chemistry concerning enantioselective synthesis has risen to meet this challenge and there now exists a plethora of approaches that can be turned to for a particular target molecule. ${ }^{1}$ On close inspection, it is apparent that the majority of these approaches are based on chemical reactions that operate via two-electron mechanisms. This is unsurprising given that the majority of mainstream organic chemistry is based on mechanisms in which electrons move in pairs. However, study of the chemistry of organic radicals has been ongoing for many years. ${ }^{2-4}$ Fundamental studies in the 1960s and 1970s progressed to powerful and practical synthetic methods in the 1980s and 1990s. The past decade has witnessed a remarkable surge in the development of new radical processes, largely driven by the popularisation and widespread embrace of photoredox catalysis. ${ }^{5-8}$ Electrochemistry is now being adopted by synthetic chemists with similar enthusiasm. ${ }^{9}$ Photoredox catalysis, in particular, has garnered so much attention because reactive open-shell intermediates with underexplored chemistries can be readily accessed using cheap LEDs together with an appropriate photocatalytic system. Numerous exciting new processes and bond disconnections have resulted, but the volume of attention has served to highlight a long-standing challenge within the broader field of radical chemistry - that of controlling enantioselectivity in processes where new stereocentres are formed.

Whilst great progress had been made in controlling diastereoselectivity in radical reactions throughout the 1980s and 1990s, dispelling the myth that radicals were too reactive to be conducive to stereocontrol, there were very few enantioselective approaches reported. ${ }^{10,11}$ The first pioneering strategies, developed by Sibi, Porter and co-workers in the late 1990s, utilized chiral Lewis acids and demonstrated that enantiocontrol was possible (Fig. 1a). ${ }^{12,13}$ They did however struggle with background reaction and as a result most protocols required stoichiometric chiral Lewis acid to obtain the highest levels of enantioselectivity. ${ }^{14}$ The widespread adoption of photoredox catalysis has focused renewed attention on this challenge. ${ }^{15}$ From the earliest days, photoredox catalysis was successfully combined with covalent modes of asymmetric organocatalysis - most prominently with chiral enamine catalysis, as first demonstrated in seminal work from Nicewicz and MacMillan (Fig. 1b). ${ }^{16}$ This closely followed an important prior development from the same group in which ceric ammonium nitrate was used as a stoichiometric single electron oxidant to generate the reactive chiral radical cation intermediate. ${ }^{17}$ Another key covalent activation mode used extensively in organocatalysis, iminium catalysis, has been impressively harnessed for the addition of radicals in more recent developments (Fig. 1c). ${ }^{18}$ Asymmetric Lewis acid catalysis has been powerfully applied in a number of different settings, including photoredox catalysis (Fig. 1d) ${ }^{19}$ and photochemistry (Fig. 1e) ${ }^{20,21}$ and the challenges of competitive background reaction have now been largely overcome. In other developments, a chiral thiyl radical has been used as a catalyst for an enantioselective radical cyclisation (Fig. 1f) (2) $^{22}$ and chiral ligands on samarium have realised stereocontrol in reductive cyclisations and cascades, albeit at stoichiometric loadings. ${ }^{23}$ Transition metal catalysis that incorporates redox processes is increasingly closely integrated into radical-based mechanisms, which offers opportunity for asymmetric induction using conventional chiral ligand strategies. ${ }^{24}$ This has been powerfully demonstrated in examples using copper, ${ }^{25-28}$ nickel, ${ }^{29-31}$ and titanium ${ }^{32,33}$ catalysis (Fig. $1 \mathrm{~g}$ ). Metalloradical-based catalysts have also emerged as powerful tools for stereocontrol in a range of radical reactions (Fig. 1h). ${ }^{34,35}$

In the key developments outlined above, the chiral catalyst is generally associated with the substrate through a covalent bond. Repulsive steric interactions at the transition state of the enantiodetermining step are primarily responsible for dictating subsequent enantioinduction, disfavouring certain pathways through destabilisation. This basic paradigm has lain at the heart of most developments in enantioselective catalysis until relatively recently and stands in contrast to the typical mechanisms by which enzymes, Nature's catalysts, control selectivity. ${ }^{36}$ At many enzyme active sites, a combination of multiple attractive non-covalent interactions act in tandem to preorganise reactants and stabilise the transition states that lead to the desired product.

Over the past several decades, synthetic chemists have come to appreciate that use of these weaker interactions in the context of carefully designed small molecule catalysts is not only viable but can be highly effective for enantiocontrol. As covalent organocatalysis has become firmly established as a distinct branch of asymmetric synthesis, the non-covalent variety has emerged as a vibrant and dynamic pursuit. ${ }^{37}$ Now, catalyst designs for two-electron processes that hinge on the most widely appreciated non-covalent interactions of hydrogen bonding ${ }^{38}$ and ion-pairing ${ }^{39-41}$ are widespread. Chiral scaffolds containing a thiourea group can be used as hydrogen bond 
donors to activate basic substrates (Fig. 2a) as well as to bind to the conjugate anions of prochiral cationic intermediates (Fig. 2b). Whilst chiral cations have been used extensively as phase transfer catalysts (Fig. 2c), chiral anion approaches have been predominantly tied to developments in asymmetric Brønsted acid catalysis. This area has been greatly advanced by the development of 1,1'-bi-2-naphthol (BINOL)-derived phosphoric acids..$^{42}$ Driven by independent reports in 2004 from the groups of Akiyama ${ }^{43}$ and Terada ${ }^{44}$ the rigid, well defined BINOL backbone, symmetrically substituted with large groups at the 3 and 3' positions, creates a C2 symmetric pocket in which the phosphoric acid functionality resides (Fig. 2d). ${ }^{45}$ These catalysts are often bifunctional; once the acidic proton has been engaged to activate basic functionality in a substrate, the catalyst's phosphate character increases i.e. it becomes more basic and can then act as a hydrogen bond acceptor to engage with the second partner in a highly organised transition state assembled through hydrogen bonds (Fig. 2e). If these catalysts are used as phosphates at the outset, they can engage in ion-pairing interactions either with a cationic reactant (Fig. 2f) or a cationic transition metal (Fig. 2g), in both cases retaining their bifunctional capability. Due to this versatility, they have been applied extensively in non-covalent approaches to enantioselective catalysis. Strategies that utilise less common non-covalent interactions such as cation- $\pi^{46}$ and anion- $\pi^{47}$ interactions and halogen bonding ${ }^{48}$ are still emerging and understanding of how to incorporate these into small molecule catalyst scaffolds is an exciting area of development. ${ }^{49,50}$ The increasing ability of density functional theory (DFT) calculations to accurately account for observed reaction outcomes provides further insight which can feedback into catalyst design. ${ }^{51,52}$ Until relatively recently there were only a handful of examples where non-covalent interactions between chiral catalyst and substrate had been utilised for inducing enantiocontrol in radical reactions. The perceived barrier of low directionality and the common label of 'weak interactions' likely led to the delay in their incorporation into enantioselective catalysts for two-electron chemistry. These preconceptions have now firmly been overcome in the two-electron world, but it is probably natural that the first wave of developments in enantioselective single-electron processes should apply longer-established covalent catalysis modes. Yet in the past few years there has been a dramatic increase in the number of successful demonstrations of non-covalent modes of catalysis being applied to this challenge, achieving outcomes not possible using other means.

This Review aims to showcase the strategies taken thus far and key developments in this still-emerging area. There are several previous reviews that discuss some aspects covered here, most relevantly from Bach and co-workers (coverage to 2014) ${ }^{53}$, Silvi and Melchiorre (coverage to 2017) ${ }^{15}$ and Lu, Zheng and co-workers (coverage to 2019). ${ }^{54}$ During preparation of this manuscript, a relevant review was published by Qiao, Zhang and co-workers. ${ }^{55}$ In some examples discussed, the actual enantiodetermining step in the mechanism may be proposed to proceed via a two-electron process and in some cases, a precise mechanistic picture may remain unconfirmed. While the major focus will be on developments in small molecule catalysts, we will also discuss recent exciting applications of enzymes to carry out enantioselective radical transformations, as the mechanisms of action in these cases surely involve attractive non-covalent interactions.

\section{Hydrogen bonding templates in photochemical reactions}

Photochemical reactions have been subject to detailed study and often include radical intermediates. The challenges of rendering such reactions enantioselective were eloquently described in the aforementioned review from Bach and co-workers. ${ }^{53}$ Early work on enantioselective synthesis in the crystalline solid state demonstrated that asymmetric induction can be achieved in photochemical reactions wherein the substrate is ion-paired with a chiral counterion as a crystalline salt, termed the "ionic auxiliary" approach. ${ }^{56}$ Remarkably, irradiation of material in a certain crystalline phase could result in high levels of asymmetric induction, an illustrative example being in the Norrish type II cyclisation of prolinol salt 1 to form chiral cyclobutanol 2 (Fig. 3a). ${ }^{57}$ In the solution phase, racemic 2 was obtained but when carried out in the solid state with material crystallised as needles, $97 \%$ enantiomeric excess (e.e.) was achieved. While intriguing, the requirement for material to be crystallised and in a specific form is a barrier to this approach becoming used more generally.

Hydrogen bonding interactions between an appropriate substrate and a chiral template have been used to powerful effect by Bach and co-workers to enable enantioselective photochemical reactions. Over the years a family of versatile templates have evolved that crucially associate with the substrate through dual hydrogen bonding interactions (Fig. 3b). ${ }^{58}$ In 2000 Bach and Bergmann first reported the intermolecular asymmetric [2+2] photocycloadditions of alkoxyquinolones such as 7 (Fig. 3c), enabled by chiral template 3 which engaged with lactam 7 through hydrogen bonding. ${ }^{59,60}$ The result was selective blocking of one prochiral face of $\mathbf{7}$ from undergoing cycloaddition after absorption of UV light. Excellent levels of enantio- and diastereocontrol were obtained and, although an excess of $\mathbf{3}$ was required to overcome racemic background reaction, this constitutes pivotal groundwork for a powerful and elegant approach, with 3 subsequently applied to a number of different reactions of lactam-containing substrates. ${ }^{58}$ It was also successfully applied to several non-photochemical radical chain reactions ${ }^{61-63}$ and, more recently, photoredox catalysis. ${ }^{64}$ In some cases high enantioselectivities were obtained, but the necessity for superstoichiometric amounts of $\mathbf{3}$ limited practicality. In efforts to overcome the need for excess template, Bach and co-workers incorporated a photoactive benzophenone unit, rendering the template bifunctional (Fig. 3b, $\mathbf{3} \rightarrow \mathbf{4}$ ). The hypothesis was that energy or electron transfer to the substrate should only occur when it is bound so the background reaction resulting from direct excitation of non-complexed substrate would be minimised and catalysis viable. This was realised for the cyclisation of an $\alpha$-amino radical, generated by photoinduced electron transfer from the pyrrolidine of the substrate $\mathbf{9}$ to the excited benzophenone unit of $\mathbf{4}$, followed by deprotonation. ${ }^{65}$ The template could be used at 30 mol\% loading, resulting in $70 \%$ e.e. (10, Fig. 3d) giving an important demonstration of the feasibility of catalysis in photochemical reactions, with hydrogen bonding interactions between substrate and catalyst proven crucial in enabling both enantioselectivity and catalytic turnover. By replacing benzophenone with xanthone as the photosensitiser component (Fig. 3b, $\mathbf{4} \rightarrow \mathbf{5}$ ), photocycloadditions could be carried out with high enantioselectivities and low catalyst loadings. ${ }^{66,67}$ A final refinement incorporated thioxanthone (Fig. 3b, 6), making the template photoactive in the visible light region, offering significant practical advantages. ${ }^{68}$ Template $\mathbf{6}$ has been used for intra- and intermolecular cycloadditions, ${ }^{69}$ and subsequently for the highly noteworthy catalytic deracemisation of allenes (Fig. 3e). ${ }^{70}$ Deracemisation processes, in which a racemate is enantioenriched without net chemical change, are extremely rare due to significant challenges related to both kinetics and 
thermodynamics. Bach and co-workers' allene deracemisation is thought to be enabled by two modes of differentiation between the enantiomers of the racemic allene 11 (Fig. 3e). The highly directional interaction of catalyst and substrate dictates the binding configurations of each enantiomer of 11. The allene substituent of $\mathbf{1 1 b}$ (tert butyl, as depicted) clashes with the thioxanthone unit when bound $(\mathbf{6} \cdot \mathbf{1 1}$ ) $)$, whilst 11a is accommodated more readily $(\mathbf{6} \cdot \mathbf{1 1 a})$. This has two consequences. Firstly, the binding coefficient of 11a with 6 was found to be significantly higher than that of 11b, so 11a preferentially occupies the catalyst. Secondly, because triplet energy transfer is highly distance dependent, the increased distance between the thioxanthone unit and the allene functionality of $\mathbf{1 1 b}$ when bound means the reaction has a lower quantum efficiency. These cooperative effects ensure that 11a preferentially undergoes racemisation via achiral triplet $\mathbf{1 2}$, whilst $\mathbf{1 1 b}$ is far more stable to further excitation. The same template has been applied to the deracemisation of cyclopropanes, albeit with moderate enantioselectivities so far. ${ }^{71}$ Overall, the breadth of reactions that have been realised using these templates clearly demonstrates the power of hydrogen bonding for bringing catalyst and substrate together in close proximity.

The thiourea functionality has been used extensively as a dual hydrogen bond donor unit in catalysts for two electron processes, including examples where these can be productively merged into tandem processes with established radical reactions. ${ }^{72}$ In 2014 Sibi, Sivaguru and co-workers demonstrated that chiral thiourea catalysts could be applied to enantioselective photochemistry. ${ }^{73}$ A thiourea was incorporated into a BINOL scaffold that also featured a phenolic hydroxy group as a second hydrogen bond donor and this bifunctional catalyst $\mathbf{1 3}$ was found to promote enantioselective intramolecular [2+2] cycloadditions of coumarins such as $\mathbf{1 2}$ (Fig. 3f). The thiourea was proposed to interact with the carbonyl of the coumarin with a second hydrogen bond occurring between the phenolic hydroxy and the $\mathrm{sp}^{3}$ hybridised oxygen of the coumarin (Fig. 3f, see box). Control experiments showed that either removal or methylation of the phenolic hydroxy resulted in very poor enantioselectivity. The network of hydrogen bonding interactions thus formed between catalyst and substrate was proposed to enable exciplex formation, which was followed by [2+2] cycloaddition, crucially in the chiral environment provided by the associated catalyst.

The final showcase of hydrogen bonding catalysis applied to enantioselective photochemistry comes from Baik, Yoon and coworkers, who have recently utilised a family of Ir-based photocatalysts that feature helical, metal-centred chirality, a strategy previously used in photoredox catalysis by Meggers and co-workers (Fig. 1d). ${ }^{19}$ Whilst Meggers' catalyst acted through Lewis acid activation, due to two vacant coordination sites, these catalysts (such as 16) incorporate a pyrazole moiety into a third ligand, which acts as a hydrogen bond donor. ${ }^{74}$ This binds the substrate and positions it appropriately to allow triplet sensitisation in the chiral environment provided by the metal complex (Fig. 3g). These catalysts were applied to intramolecular cycloadditions of substrates such as $\mathbf{1 5}$, in which generally high enantiomeric excesses (up to $91 \%$ ) could be achieved. ${ }^{75}$ Interestingly, careful NMR titration studies to establish the association of substrate with pyrazole suggested that additional substrate-ligand interactions were occurring with the aryl ring of one of the cyclometalated arylpyridine ligands (Fig. 3g, see box). Binding was increased by tuning the electronics of the aforementioned aryl ring and DFT calculations suggested that a ligand-substrate $\pi-\pi$ interaction is making an important contribution to enantioinduction in addition to two hydrogen bonds between the lactam and the pyrazole. This ligand was then successfully applied to intermolecular [2+2] cycloadditions using maleimide as a partner, in which generally superior enantioselectivities were obtained. ${ }^{76}$ Detailed mechanistic studies suggest that triplet energy transfer occurs from the catalyst to the maleimide, rather than to the bound substrate. This contrasts with the earlier intramolecular example and demonstrates that ground-state pre-association between the chiral catalyst and the sensitised substrate is not necessary to obtain high enantioselectivity. Overall, this work further underscores the advantage of attractive noncovalent interactions drawing the substrate in close to the chirality of a bifunctional catalyst, which can then promote reactivity in that dedicated environment.

\section{Dual hydrogen bond donor catalysts and chiral cations}

The unique ability of dual hydrogen bond donor catalysts to assemble transition states was impressively combined with photoredox catalysis in 2015 by Ooi and co-workers, who reported an enantioselective synthesis of 1,2-diamines via the redox-neutral coupling of aldimines (18) with $N$-arylaminomethanes (19) (Fig. 4a). ${ }^{77}$ An iridium-based photoredox catalyst was used alongside chiral phosphonium salt catalyst 21. The proposed mechanism commences with single electron oxidation of the amine in 19, which forms a neutral $\alpha$-amino radical after deprotonation. The reduced photocatalyst itself reduces the aldimine 18, forming a radical anion that ion exchanges with the cationic phosphonium salt. A challenging, enantiodetermining radical-radical coupling (Fig. 4a, 22) is then proposed to ensue under the careful control of the chiral phosphonium cation, which most likely engages in highly directional dual hydrogen bonding interactions with the radical anion, in addition to electrostatic interactions arising from the opposite charges. A later study suggests that the precise sequence of redox events is not critical to productive enantioselective coupling, as a catalytic cycle initiated by oxidative quenching of an excited photosensitiser was also viable. ${ }^{78}$

Chiral cations derived from cinchona alkaloids have been used extensively for control in two-electron processes, most prominently in asymmetric phase-transfer organocatalysis. ${ }^{79}$ Overtly, an ion pairing interaction is the key attractive interaction in the transition state for such reactions, but it is widely accepted that multiple weaker hydrogen bonding donor interactions from the cation play a crucial role in geometry control. ${ }^{80}$ These occur from the $\mathrm{C}-\mathrm{H}$ bonds adjacent to the ammonium cation, which are significantly acidified due to inductive effects, as well as in some cases the hydroxy O-H. In 2015, Melchiorre and co-workers demonstrated that chiral cations such as 28 can be effective for control in a radical reaction with a highly enantioselective perfluoroalkylation of $\beta$-ketoesters (Fig. 4b). ${ }^{81}$ In this process, a prochiral enolate ion-pairs with the chiral cinchonine-derived cation in solution. This forms a coloured electron donoracceptor complex 25 with a perfluoroalkyl iodide that, upon irradiation with white LEDs, undergoes electron transfer to generate a perfluoroalkyl radical. This intermediate then reacts with another ion-paired enolate in the enantiodetermining step (24 $\rightarrow \mathbf{2 6}$ ). The ketyl radical $\mathbf{2 6}$ thus formed abstracts an iodine atom from the perfluoroalkyl iodide in chain propagation, furnishing $\mathbf{2 7}$ after heterolytic C-I cleavage. A recent DFT study provides support for a network of hydrogen bonding interactions between the cation and anion being 
responsible for the high selectivity in this process. ${ }^{82}$ Given how widely privileged chiral cations such as these have been used in twoelectron organocatalysis, it seems likely that further developments using related approaches can be expected in the future.

Jiang and co-workers reported an enantioselective reduction of diketones (such as 29) and ketimines thought to proceed via a ketyl radical, in which asymmetry is induced by the dual hydrogen bond donor guanidinium salt 32 (Fig. 4c)..$^{83}$ Electron transfer is proposed to occur from the reduced dicyanopyrazine photocatalyst $\mathbf{3 1}$ to form a ketyl radical anion, which interacts strongly with the dual hydrogen bond donor organocatalyst. A further single electron reduction gives an $\alpha$-oxyanion still associated with the chiral catalyst and it is the latter that mediates the enantiodetermining protonation to form the product 33. Similar outcomes were observed in many cases in the absence of photocatalyst and it was proposed that a photochemically active electron donor-acceptor complex between $\mathbf{2 9}$ and the stoichiometric amine accounts for this. The same authors later used a similar catalytic system to enable an enantioselective dehalogenative protonation reaction. ${ }^{84}$

\section{Chiral Brønsted acids and bases}

Asymmetric Brønsted acid catalysis is a hugely important branch of organocatalysis and bulky BINOL-derived phosphoric acids have been instrumental to this success (Fig. 2d-g). Whilst hundreds of studies have utilised chiral Brønsted acids in two electron processes, successful application to radical reactions has been slow on the uptake but is now accelerating rapidly. Whilst an early report from Lee and Kim showed that promising enantioselectivities could be achieved in the addition of alkyl radicals to imines using 30 mol\% of a chiral $N$-triflyl phosphoramide catalyst, ${ }^{85}$ it was a breakthrough paper from Knowles and co-workers in 2013 that demonstrated how powerful the BINOL-phosphoric acid scaffold could be for enantioselective catalysis of radical processes. Here the scaffold was used alongside an Ir photoredox catalyst to enable a highly enantioselective aza-pinacol cyclisation to give products such as 43 (Fig. 5b). ${ }^{86}$ The activation mode exploits proton-coupled electron transfer (PCET) to perform otherwise endergonic single electron reductions of aryl-alkyl ketones. Pre-activation of the ketone $\mathbf{4 2}$ towards PCET is achieved via a hydrogen-bonding interaction between the phosphoric acid catalyst and the ketone, priming it for concerted proton transfer from the acid and single-electron reduction by the photocatalyst. The neutral ketyl radical formed thereafter is poised for enantiodetermining cyclisation and is crucially able to remain associated with the chiral phosphate anion (produced following PCET) throughout this process, by way of hydrogen bonding interactions (44). This report was highly influential as it demonstrated that even an apparently single hydrogen bond between the reactive radical intermediate and a chiral catalyst is sufficient to induce high levels of asymmetry.

In 2018, Phipps and co-workers demonstrated how asymmetric Brønsted acid catalysis could be applied to achieve enantioselectivity in a very well-established class of radical reaction - Minisci-type alkylation of heteroarenes (Fig. 5c). ${ }^{87}$ Their approach took into consideration the typical requirement for Minisci-type reactions to be carried out in the presence of acid, which lowers the heteroarene LUMO and permits nucleophilic radicals to attack the $\pi$-system. Using a BINOL-phosphoric acid, the hypothesis was that if the alkyl radical possessed a hydrogen bond donor, a network of attractive non-covalent interactions may be achieved during the process, allowing enantiocontrol. Photoredox catalysis was used to drive the transformation, which operated effectively on a range of quinolines and electron-deficient pyridines. The mechanistic proposal involves $N$-acyl $\alpha$-aminoalkyl radicals, generated by decarboxylative fragmentation of an $\mathrm{N}$-acyloxyphthalimide-based redox-active ester (46) after single electron reduction from the photocatalyst, adding reversibly to the protonated heteroarene (48 to $\mathbf{4 9}$ ). A kinetic isotope effect experiment suggested that deprotonation of the subsequently formed radical cation $\mathbf{4 9}$ is likely to be the enantiodetermining step. Hydrogen bonding interactions with the incoming radical were crucial, as demonstrated by poor e.e. when a proline-derived radical, bearing no hydrogen bond donor, was employed. In collaboration with Sigman and Reid, the same authors embarked on a data-driven, statistical approach ${ }^{88}$ to develop a predictive model for the developed reaction. ${ }^{89}$ Multivariate linear regression analysis of a targeted dataset comprising different substrates and catalysts led to a model that was able to effectively transfer enantioselectivity outcomes to new reaction components: in this case pharmaceuticallyrelevant diazines such as pyrimidines and pyrazines, which had not been explored in the original report.

In related developments, Jiang and co-workers communicated a very similar approach, using photocatalyst $\mathbf{3 1}$ and 1,1'-spirobiindane7,7'-diol (SPINOL)-derived phosphoric acids, in which good enantioselectivities were achieved on isoquinolines, a class of substrate that gave low e.e. under the conditions used by Phipps and co-workers. ${ }^{90}$ Zheng and Studer demonstrated that the previously-developed catalytic approach could be applied to a three-component reaction wherein the requisite $\alpha$-aminoalkyl radical is efficiently assembled from the reaction of an alkyl radical with an $N$-acyl enamide. ${ }^{91}$

Also in 2018, Jiang and co-workers reported several reactions in which a chiral phosphoric acid is proposed to control enantioselectivity in the coupling of two radicals by concurrently engaging both radical intermediates via hydrogen bonding. One notable example is the enantioselective, decarboxylative coupling between 1,2-diketones and $\mathrm{N}$-arylglycines (Fig. 5d). ${ }^{92}$ Single-electron oxidation of the nitrogen atom of $\mathbf{5 1}$ by the photoredox catalyst is followed by deprotonation and decarboxylation to form a stabilised $\alpha$-amino radical. The reduced photocatalyst is then proposed to reduce the 1,2-diketone $\mathbf{5 0}$ to a ketyl radical. Hydrogen bonding interactions between chiral catalyst and the two radicals are proposed to account for the high levels of asymmetric induction in the transition state (53), although full details of the origin of selectivity are yet to be determined. This strategy was also applied to the asymmetric reaction between racemic $\alpha$-bromoketones and $N$-aryl glycine derivatives ${ }^{93}$ and later further expanded to 3chlorooxindoles. ${ }^{94}$ In all cases similar ternary transition states are proposed in which the chiral phosphoric acid orchestrates enantiodetermining radical-radical coupling. The Jiang group have demonstrated further impressive advances using the same catalytic system, which showcases how powerful phosphoric acid catalysts are for orchestrating a variety of challenging radical reactions in an enantioselective sense. Building on previous work in which non-enantioselective radical addition was followed by enantiodetermining protonation, ${ }^{95}$ they showed that vinyl pyridines such as $\mathbf{5 4}$ are amenable to enantiodetermining radical conjugate addition of prochiral ketyl and $\alpha$-aminoalkyl radicals to give products such as $\mathbf{5 7}$ (Fig. 5e). ${ }^{96}$ In this step it is presumed that the chiral phosphate engages in hydrogen bonding interactions with both components, leading to a highly organised transition state (58). The resultant $\alpha$-pyridyl radical 
is proposed to be quenched through hydrogen atom transfer (HAT) or consecutive electron and proton transfers from the dihydropyridine reductant 56. In a remarkable display of versatility, the reaction scope encompasses prochiral radicals generated from benzophenones, benzaldehydes, aldimines and ketimines and it is notable that the stereocentre generated is quite some distance from the pyridine nitrogen.

In the examples surveyed so far, the catalyst has been used in its native acid form to perform some type of Brønsted acid activation, although the phosphate form is often postulated to come into play later in the mechanism. In an interesting study, Nicewicz and coworkers sought to use chiral phosphates from the outset as counterions to highly oxidising, cationic photoredox catalysts. The hypothesis was that ion pairing of the chiral anion with the radical cation generated on single electron oxidation of an alkene may allow enantioinduction in an ensuing cation radical Diels-Alder reaction. The enantioselectivities (up to 50\% e.e.) were moderate but encouraging, particularly since little other functionality was present in the substrates to aid in transition state assembly. ${ }^{97}$

In a strategy also involving the basic phosphate from the outset, Knowles and co-workers took a bioinspired approach to the asymmetric enantioselective synthesis of pyrroloindolines via a radical mechanism (Fig. 6a). ${ }^{98}$ They imagined a process in which the chiral phosphate base assists in oxidative PCET on an indole substrate such as 59, resulting in indole radical cation 61. Crucially, the phosphate should remain associated through electrostatic and hydrogen-bonding interactions throughout a subsequent enantiodetermining radical-radical cross-coupling of the tryptophanyl radical cation with the persistent radical (2,2,6,6tetramethylpiperidin-1-yl)oxyl (TEMPO). This strategy worked extremely well, resulting in the pyrroloindoline products $\mathbf{6 0}$ with excellent control of enantioselectivity. Because TEMPO-H was observed to inhibit the reaction, most likely by preferentially undergoing undesired oxidative PCET, the hypervalent iodine reagent TIPS-EBX was found to be necessary as a net oxidant, with the carboxylate generated after reduction acting as the terminal base. Detailed mechanistic studies provided strong support for the proposed oxidative PCET pathway and protocols were developed for further functionalisation of these isolated TEMPO adducts via mesolytic cleavage of the weak $\mathrm{C}-\mathrm{O}$ bond. This was achieved using photoredox catalysis, involving single electron oxidation to give configurationally biased cation 63, which can undergo stereoselective trapping with a range of nucleophiles. The approach was showcased in concise asymmetric syntheses of several dimeric pyrroloindoline natural products, such as (-)-calycanthidine (62).

As previously discussed, deracemisation is a formidable challenge in enantioselective catalysis, with few strategies able to accomplish it. In the course of targeting asymmetric hydroamination reactions through a PCET strategy, Knowles, Miller and co-workers developed a remarkable deracemisation of ureas such as $\mathbf{6 4}$ (Fig. 6b) ${ }^{99}$ Upon exposure of $\mathbf{6 4}$ to visible light with an Ir photoredox catalyst, a chiral phosphate and thiophenol, modest enantioenrichment of recovered $\mathbf{6 4}$ was observed. Envisioning that this may occur by single electron oxidation, enantioselective deprotonation of the resulting radical cation by the chiral phosphate and subsequent nonselective HAT to reform the starting material, the authors speculated whether a chiral thiol may have the ability to perform the terminating HAT in an enantioselective manner. The resulting combination of chiral phosphate salt $(S)$-36-Bu $\mathrm{H}_{4} \mathrm{~N}$ and peptide-derived thiol 65, each playing their allotted role at different points in the mechanism, resulted in excellent levels of enantioselectivity. Detailed studies of the enantioselectivity induced by each chiral catalyst alone enabled the net enantioselectivity resulting from the two chiral catalysts working in tandem to be predicted. The predicted enantioselectivities matched closely to the experimental results, demonstrating the synergistic manner in which the two catalysts operate. The proposed mechanism begins with the excited photocatalyst indiscriminately oxidising 64 to give radical cations $(R)-\mathbf{6 6}$ and $(S)-\mathbf{6 6}$, the latter of which is selectively deprotonated by the chiral phosphate to form achiral radical 67. Enantioselective HAT to 67 from the chiral thiol 65 preferentially forms $(R)$-64. Combined together, this results in a ratio of $(R)$-64:(S)-64 of up to 95:5 in the reisolated material and is a remarkable example of the kinetic decoupling of $\mathrm{C}-\mathrm{H}$ bond breakage and formation. The free $\mathrm{N}-\mathrm{H}$ of the pendant amide group was found to be important in enhancing enantioselectivity in the deprotonation step, suggesting that there could be important hydrogen bonding interactions with the chiral phosphate during this process. Conversely, this free $\mathrm{N}-\mathrm{H}$ was found to be unimportant for the enantioselective HAT step, meaning that thiol catalyst 65 is able to perform enantioselective HAT to radical 67, which is uncharged and has few overt handles for interaction, a particularly notable achievement.

We have previously discussed Knowles' influential aza-pinacol cyclisation, where enantioselectivity is controlled by a hydrogen bond between a ketyl radical and a chiral phosphate after PCET reduction (Fig. 5b). In an important recent contribution the same group targeted enantioselective hydroamination, and sought to generate an $N$-centred sulfonamidyl radical through PCET oxidation, with aid of a chiral phosphate base (Fig. 6c). ${ }^{100}$ The crucial question was whether the phosphoric acid formed would remain associated with the neutral sulfonamidyl radical during enantiodetermining cyclisation (Fig. 6c, inset box); very little information is known on the basicity of sulfonamidyl radicals and their related proficiency as hydrogen bond acceptors. This work highlights the challenges in associating chiral catalysts with neutral free radicals - the putative enantiodetermining step in many examples discussed in this section feature a radical intermediate that is charged, enabling ion-pairing interactions with the catalyst, or bears a strong hydrogen bond donor. Overcoming these design constraints is key to broadening the scope of enantioselective radical reactions. Following optimisation of catalyst structure, excellent enantiocontrol was achieved in products such as $\mathbf{6 9}$ utilising triazolium-substituted BINOL-derived phosphate 38 together with a bulky thiol to engage in terminal HAT post-cyclisation. These results, as well as various mechanistic experiments, supported hydrogen bonding interactions between catalyst and substrate being crucial during the cyclisation. This study provides another powerful example of a way in which the BINOL-phosphoric acid scaffold can be used to associate with reactive radical intermediates.

\section{Approaches incorporating transition metals}

None of the approaches covered thus far have involved transition metals operating in redox processes, apart from as external photoredox catalysts. As touched upon in the introduction, transition metal catalysis is increasingly being harnessed in radical-based mechanisms and deployment of conventional chiral ligand strategies to render these asymmetric has had significant success. Several transition metal-based strategies in which attractive non-covalent interactions feature prominently in the likely modes of stereoinduction 
will be discussed herein. We believe they provide instructive examples of how non-covalent interactions can be closely integrated with versatile transition metal reactivity and that there is much to explore in this line of inquiry.

In Nature, the oxidation of aliphatic C-H bonds by cytochrome P450 enzymes is well established to proceed via a radical pathway involving a "radical rebound" sequence mediated by iron. ${ }^{101,102}$ The development of selective C-H oxidation reactions is an important challenge for synthetic chemists and there have been several examples which seek to emulate this in an enantioselective manner using ligand-substrate non-covalent interactions. In 2015, Bach and co-workers grafted a Ru-porphyrin onto their proven chiral template, which had been so effective for enantioselective photochemistry (Fig. 3). This enabled the highly enantiotopo-selective $\mathrm{C}-\mathrm{H}$ oxygenation of spirocyclic oxindoles such as $\mathbf{7 0}$ (Fig. 7a). ${ }^{103}$ By tethering the chiral lactam functionality to the porphyrin ligand (72), substrate-ligand association via dual hydrogen bonds could direct reactivity. This association was shown to be crucial not only for enantioselectivity but also catalyst activity, as demonstrated by $N$-methylation studies of substrate and ligand. 2,6-Dichloropyridine- $N$ oxide (71) served as an external oxidant to generate the reactive Ru-oxo complex. Careful orientation of the substrate relative to the metal centre facilitated selective HAT from one of two enantiotopic methylene groups on the oxindole, followed by hydroxy group transfer through the radical rebound mechanism. Evidence for selectivity-determining HAT was provided by experimental and computational studies.

This elegant approach to chiral ligand design paved the way for the same group to develop subsequent enantioselective benzylic hydroxylations of 3,4-dihydroquinolones (such as 74) and 3-substituted quinolones, in which overoxidation to the ketone was avoided by exchanging ruthenium for manganese (Fig. 7b). ${ }^{104,105}$ Once again, chiral ligand 72 provided the requisite enantiofacial discrimination for selective HAT and subsequent hydroxylation. Furthermore, the strong directing ability of the dual hydrogen bonding system enabled inherent site-selectivity to be overridden in some substrates (e.g. doubly benzylic $\mathbf{7 4}$ - alternative site shown in red).

The use of chiral phosphates as counterions for cationic transition metal catalysts has been extensively explored, but the majority of these advances concern two electron processes. ${ }^{39-41}$ Copper is a versatile transition metal that frequently engages in radical mechanisms and, whilst there are established ligands for copper that largely exert stereocontrol through steric repulsion, ${ }^{25-28}$ there are examples emerging of chiral phosphate ligands (or counterions, depending on the degree of association) for copper that are highly adept at inducing enantiocontrol in reactions involving radical intermediates. Liu and co-workers have developed a number of such transformations likely involving crucial attractive non-covalent interactions between substrate and phosphate. ${ }^{106}$ In one example, they developed an enantioselective aminotrifluoromethylation of alkenes such as 76 (Fig. 7c). ${ }^{107}$ Copper (I) is proposed to catalytically generate, from reagent 78, a trifluoromethyl radical that undergoes addition to 76, giving achiral radical 79. Enantioinduction is proposed to occur via one of two possible mechanisms: through addition of $\mathrm{Cu}(\mathrm{II})$ phosphate to $\mathbf{7 9}$ to generate a chiral copper (III) intermediate (80), wherein the pendant urea moiety engages in hydrogen bonding with the copper-associated chiral phosphate, or through ion pairing with carbocation 81, generated via oxidation of the radical adduct by copper (II). They favour the first scenario and invoke a similar mechanism involving a $\mathrm{Cu}(\mathrm{III})$ intermediate in their subsequently developed radical diamination, which uses $N$-acyloxyamines as precursors to nitrogen-centred radicals to functionalise similar scaffolds. ${ }^{106,108}$ The group also extended their alkene aminofunctionalisation to an amination of allylic and benzylic $\mathrm{C}-\mathrm{H}$ bonds via hydrogen atom abstraction, with a chiral $\mathrm{Cu}(\mathrm{II})$ phosphate complex proposed to intercept the prochiral radical intermediate, as described above, with the assistance of hydrogen bonding. ${ }^{109}$ Very recently, they showed that the Cu-phosphate system can be used to desymmetrise 1,3-diols such as $\mathbf{8 2}$ by a cyclising alkene difunctionalisation (Fig. 7d). ${ }^{110}$ DFT calculations suggest that the initially formed radical adduct (analogous to 79) is trapped by $\mathrm{Cu}$ (II) phosphate, but the resulting $\mathrm{Cu}(\mathrm{III})$ intermediate rapidly collapses to a carbocation. This is trapped by a hydroxy group in the enantiodetermining step, in which multiple hydrogen bonding contacts between substrate and chiral phosphate are invoked (85). Mechanistic questions still remain in these processes but they convey the strong message that chiral phosphate counterions could produce very exciting outcomes when partnered with transition metals that can engage in radical mechanisms, leading to unique combinations of reactivity (from the metal) and selectivity (from the counterion). Copper is one such promising candidate, but the rich radical chemistry undergone at nickel metal centres surely offers much potential in this regard.

\section{Biocatalytic approaches to non-natural radical reactions}

The unique potential that enzymes possess to catalyse challenging chemical transformations with the highest levels of efficiency and stereocontrol makes their application to enantioselective synthesis very appealing. ${ }^{111,112}$ Major advances in protein engineering combined with the increased awareness of the opportunities presented by substrate promiscuity have unleashed new catalytic activities between enzymes and non-natural substrates. ${ }^{113-117}$ It is well established that enzymes are adept at mediating radical transformations in Nature. ${ }^{118-}$

${ }^{120}$ In many cases, it is likely the restricted active site of the protein 'shields' the reactive radical from deleterious side reactions in addition to guiding the path of the desired transformation, commonly through the action of multiple non-covalent interactions. If biocatalytic approaches could be developed to perform non-natural radical reactions in an enantioselective manner, this could potentially have a sizable impact on enantioselective synthesis, given that chemical approaches are still relatively limited. Recently, the Hyster group has been at the forefront of developing such strategies and several examples are surveyed in this Review as the enantioinduction is almost certainly controlled by attractive non-covalent interactions at the enzyme active site.

In 2016, Hyster and co-workers reported an enantioselective dehalogenation of racemic lactones, such as $\mathbf{8 6}$ and $\mathbf{8 7}$, which was achieved by photoexcitation of nicotinamide-dependent ketoreductases (KREDs) (Fig. 8a). ${ }^{121}$ Photoexcitation enables the enzyme's cofactor nicotinamide adenine dinucleotide phosphate (NADPH) - typically a hydride donor - to function instead as a powerful single electron reductant. ${ }^{122}$ Within the enzyme's active site, a charge transfer complex comprised of substrate and NADPH is formed (90). Control experiments confirmed that charge-transfer complex formation is reliant on the presence of the enzyme. Furthermore, it was shown that the KRED increased the fluorescence lifetime of NADPH by more than 20-fold. Taken together, these results demonstrate that binding of substrate and co-factor within the enzyme active site, likely via multiple non-covalent interactions, is crucial to the viability of the process. Upon visible light irradiation electron transfer from NADPH ensues and debromination by a spin-centre shift 
mechanism gives a prochiral radical $(\mathbf{9 0} \rightarrow \mathbf{9 1})$. This undergoes enantiodetermining HAT from the radical cation $\mathrm{NADPH}^{\cdot+}$ to form the product $(\mathbf{9 1} \rightarrow \mathbf{9 2})$. The positioning of the prochiral radical in the enzyme active site is crucial for exerting control over the facial selectivity of hydrogen atom delivery. This is enabled by hydrogen bonding interactions between the substrate and amino acid residues at the enzyme active site, delivering the products with high enantioselectivity. Indeed, modelling studies involving lactone (S)-89 and one of the KREDs, RasADH, displayed key hydrogen bonding interactions between tyrosine and serine residues of the enzyme active site and the carbonyl oxygen of the substrate. Experimental observations combined with docking models showed that the enzymes did not discriminate between enantiomers of the $\alpha$-bromolactone substrates for binding, highlighting that a mechanism involving kinetic resolution of the racemic substrates was not operative. Different KREDs were identified which could provide access to both the $R$ and $S$ enantiomers of the products in good yields and enantioselectivities although the requirement for formation of a charge transfer complex limited the scope to lactone substrates. Subsequently, a related system that combines the use of a nicotinamide-dependant double bond reductase (DBR) with the organic dye photocatalyst Rose Bengal (RB) was developed for the enantioselective radical deacetoxylation of tetralones such as $\mathbf{9 3}$ (Fig. 8b). ${ }^{123}$ Within the enzyme's active site, the substrate associates with a tyrosine residue via a hydrogen bond, a known mode of binding for this enzyme family, and remains associated throughout (95). This hydrogen bond is not only crucial for enabling enantiocontrol in the process but also alters the redox potential of $\mathbf{9 3}$, facilitating an otherwise endergonic single electron transfer by the reduced photocatalyst $\left(\mathrm{RB}^{\cdot}{ }^{-}\right)$. The prochiral radical 96 is subsequently quenched via enantiodetermining HAT from the NADPH co-factor present in the active site, akin to their previous work (vide supra). This hydrogen bond-mediated 'enzymatic redox activation' of the carbonyl has been further extended to flavin (FMN)-dependent ene-reductases (EREDs) where the enantioselective reduction of acetophenones was achieved using the common photocatalyst $\mathrm{Ru}(\mathrm{bpy})_{3} \mathrm{Cl}_{2}{ }^{124}$ Most recently, a similar system was successfully applied to the enantioselective reduction of vinyl pyridines. ${ }^{125}$

Their previous enantioselective dehalogenation of racemic lactones had hinged on the formation of a charge transfer complex between the substrate and NADPH to initiate the process (Fig. 8a). To move away from this limitation, Hyster and co-workers developed a protocol that used an engineered flavin-dependent ERED, crucially enabling flavin hydroquinone $\left(\mathrm{FMN}_{\mathrm{hq}}\right)$ to act as a single electron reductant and acyclic $\alpha$-bromoesters such as $\mathbf{9 7}$ to become viable substrates (Fig. $8 \mathrm{c}$ ). ${ }^{126}$ Once within the enzyme's active site $\mathrm{FMN}_{\mathrm{hq}}$ possesses increased reducing ability (compared to NADPH) and is thus capable of directly reducing the enzyme-bound $\alpha$-bromoester substrates, alleviating the requirement for photoexcitation in this reaction. Bound to the enzyme active site via hydrogen bonds to histidine and asparagine, dehalogenation of the substrate occurs following electron transfer from $\mathrm{FMN}_{\mathrm{hq}}$, furnishing an enzyme-bound prochiral $\alpha$-acyl radical which selectively abstracts a hydrogen atom from flavin semiquinone $\left(\mathrm{FMN}_{\mathrm{sq}}\right)(\mathbf{9 9})$. During optimisation the authors hypothesised that a tyrosine residue present in the active site of the native enzyme, on the opposite face of the prochiral radical (relative to $\mathrm{FMN}_{\mathrm{sq}}$ ), may serve as an alternative hydrogen source and thus reduce the enantioselectivity of the reaction. Crucially, replacement of that tyrosine residue with a phenylalanine via site-selective mutagenesis improved the enantioselectivity of the reaction from $76 \%$ e.e. with the native enzyme (GluER), to 94\% e.e. with the Y117F variant (99).

Hyster and co-workers have recently extended the capabilities of FMN-dependent EREDs to enable the highly enantio- and diastereoselective cyclisation of $\alpha$-chloroacetamides such as $\mathbf{1 0 0}$ to lactams, a reaction that would be extremely challenging to control using existing methods for chemical catalysis (Fig. 8d). ${ }^{127}$ Supported by UV-vis analysis, it was proposed that in order for the desired electron transfer to proceed, the binding of substrate $\mathbf{1 0 0}$ (via hydrogen bonding) and $\mathrm{FMN}_{\mathrm{hq}}$ within the enzyme's active site were critical to enabling the formation of a charge transfer complex, otherwise no reactivity was observed. Photoexcitation of a FMN $\mathrm{h}_{\mathrm{hq}}$-substrate charge transfer complex facilitates single electron reduction of $\mathbf{1 0 0}$ to form an $\alpha$-acetamide radical. X-ray crystallographic data of the wild-type GluER and mutant GluER-T36A enzymes showed canonical histidine and asparagine binding residues in the active site and the authors propose the $\alpha$-acetamide radical is bound to these residues through hydrogen bonding and held in a conformation that renders cyclisation highly favourable. The enzyme controlled both the facial selectivity of the radical cyclization (102) and the subsequent delivery of the hydrogen atom to the exocyclic radical (103). This elegant study demonstrates the truly unique capabilities of enzymes to exert stereocontrol in the most challenging of transformations. Very recently, the same authors reported an enantioselective synthesis of oxindoles from racemic $\alpha$-haloamides using a related strategy, highlighting the versatility of FMN-dependent EREDs for enabling challenging enantioselective radical chemistry. ${ }^{128}$

\section{Concluding remarks and future prospects}

This Review has summarised the development of strategies that utilise attractive non-covalent interactions to control enantioselectivity in reactions involving radical intermediates. Undoubtedly, much of the recent progress in enantioselective radical chemistry in general has been spurred by advancements in photoredox catalysis. The power of harnessing catalyst-substrate non-covalent interactions to control highly reactive intermediates was showcased early on in the context of photochemical reactions and a number of different catalyst scaffolds have since been shown amenable. As the examples above demonstrate, in the era of photoredox catalysis, Brønsted acids, bases, hydrogen bond donors and ion pairing catalysts have all been utilised successfully to varying extents. Reflecting upon the most widely used modes in non-covalent enantioselective catalysis (Fig. 2), it is notable that they are now all represented to some degree. It is evident from the examples covered that one particular scaffold stands out in terms of its breadth of application - the BINOL-derived phosphoric acid, whose conjugate base is also a key player in many mechanisms. The versatility of this privileged, bifunctional scaffold is widely appreciated in ionic asymmetric chemistry and it has proved itself powerful in numerous diverse contexts. Perhaps that privileged status has made it an early instrument for exploring the perceived greater challenge of asymmetric radical chemistry. Chiral template catalysts that use rigid hydrogen bonding to precisely poise their reactive sites close to a particular site on the substrate have proved effective and offer tantalising glimpses into the future exertion of both positional and stereochemical control in radical processes. Whilst it is true that in most cases the radical intermediate engaging in the enantiodetermining step possesses either 
a charge or suitable functionality with which to engage the catalyst, given the broad range of common functional groups that are capable of participating in non-covalent interactions, this seems unlikely to become a major limitation.

Whilst the examples in this review have all by-and-large utilised ion pairing and hydrogen bonding interactions, the diversity of noncovalent interactions that are now being integrated into catalyst designs for two-electron processes will surely filter down in the future and be applied to radical processes. One might anticipate that this could actually happen rather quickly, since the basic understanding and appreciation of how to integrate such interactions into small molecule scaffolds in ionic processes, as well as the understanding of how transition states may be arranged from DFT studies, should aid and expedite progress. It seems inevitable too that the push to apply novel biocatalysis strategies to address synthetic chemistry challenges will result in rapid further progress in the stereocontrol of radical processes. We hope that this Review will further stimulate collective thinking on new approaches for applying non-covalent modes of catalysis to the challenges that lie in the control of enantioselectivity when dealing with radical reactions.

\section{Acknowledgements}

R.S.J.P. is grateful to GlaxoSmithKline and the EPSRC for a CASE PhD studentship. We are grateful to the Leverhulme Trust for a research grant (RPG-2018-081), which provided funding for A.C.C. R.J.P. is grateful to the Royal Society for a University Research Fellowship and the ERC for a starting grant (StG 757381, NonCovRegioSiteCat).

\section{Author contributions}

R.S.J.P and R.J.P. conceived and planned the article and R.S.J.P, A.C.C. and R.J.P wrote the manuscript.

\section{Competing Interests}

The authors declare no competing interests.

Ojima, I. Catalytic Asymmetric Synthesis 3rd edn, (Wiley-VCH, Weinheim, 2010).

Renaud, P. \& Sibi, M. P. Radicals in Organic Synthesis. (Wiley-VCH, Weinheim, 2001).

Zard, S. Z. Radical Reactions in Organic Synthesis. (Oxford University Press, Oxford, 2003).

Togo, H. Advanced Free Radical Reactions for Organic Synthesis. (Elsevier, Amsterdam, 2004).

Narayanam, J. M. R. \& Stephenson, C. R. J. Visible light photoredox catalysis: applications in organic synthesis. Chem. Soc. Rev. 40, 102-113 (2011).

Schultz, D. M. \& Yoon, T. P. Solar synthesis: prospects in visible light photocatalysis. Science 343, 1239176 (2014).

Shaw, M. H., Twilton, J. \& MacMillan, D. W. C. Photoredox Catalysis in Organic Chemistry. J. Org. Chem. 81, 6898-6926 (2016).

Romero, N. A. \& Nicewicz, D. A. Organic Photoredox Catalysis. Chem. Rev. 116, 10075-10166 (2016). Yan, M., Kawamata, Y. \& Baran, P. S. Synthetic Organic Electrochemistry: Calling All Engineers. Angew. Chem. Int. Ed. 57, 4149-4155 (2018).

Porter, N. A., Giese, B. \& Curran, D. P. Acyclic stereochemical control in free-radical reactions. Acc. Chem. Res. 24, 296-304 (1991).

Curran, D. P., Porter, N. A. \& Giese, B. Stereochemistry of Radical Reactions: Concepts, Guidelines and Synthetic Applications. (Wiley-VCH, Weinheim, 1995).

Sibi, M. P., Ji, J., Wu, J. H., Gürtler, S. \& Porter, N. A. Chiral Lewis Acid Catalysis in Radical Reactions: Enantioselective Conjugate Radical Additions. J. Am. Chem. Soc. 118, 9200-9201 (1996).

Sibi, M. P. \& Porter, N. A. Enantioselective Free Radical Reactions. Acc. Chem. Res. 32, 163-171 (1999).

Sibi, M. P., Manyem, S. \& Zimmerman, J. Enantioselective Radical Processes. Chem. Rev. 103, 3263-3296 (2003).

Silvi, M. \& Melchiorre, P. Enhancing the potential of enantioselective organocatalysis with light. Nature 554, 4149 (2018).

Nicewicz, D. A. \& MacMillan, D. W. C. Merging Photoredox Catalysis with Organocatalysis: The Direct Asymmetric Alkylation of Aldehydes. Science 322, 77-80 (2008).

Beeson, T. D., Mastracchio, A., Hong, J.-B., Ashton, K. \& MacMillan, D. W. C. Enantioselective Organocatalysis Using SOMO Activation. Science 316, 582-585 (2007).

Murphy, J. J., Bastida, D., Paria, S., Fagnoni, M. \& Melchiorre, P. Asymmetric catalytic formation of quaternary carbons by iminium ion trapping of radicals. Nature 532, 218-222 (2016).

9 Huo, H. et al. Asymmetric photoredox transition-metal catalysis activated by visible light. Nature 515, $100-103$ (2014).

Brimioulle, R. \& Bach, T. Enantioselective Lewis Acid Catalysis of Intramolecular Enone [2+2]

Photocycloaddition Reactions. Science 342, 840-843 (2013). 
Du, J., Skubi, K. L., Schultz, D. M. \& Yoon, T. P. A Dual-Catalysis Approach to Enantioselective [2 + 2] Photocycloadditions Using Visible Light. Science 344, 392-396 (2014).

22 Hashimoto, T., Kawamata, Y. \& Maruoka, K. An organic thiyl radical catalyst for enantioselective cyclization. Nat. Chem. 6, 702-705 (2014).

23 Kern, N., Plesniak, M. P., McDouall, J. J. W. \& Procter, D. J. Enantioselective cyclizations and cyclization cascades of samarium ketyl radicals. Nat. Chem. 9, 1198-1204 (2017).

24 Twilton, J. et al. The merger of transition metal and photocatalysis. Nat. Rev. Chem. 1, 0052 (2017).

25 Liwosz, T. W. \& Chemler, S. R. Copper-Catalyzed Enantioselective Intramolecular Alkene Amination/Intermolecular Heck-Type Coupling Cascade. J. Am. Chem. Soc. 134, 2020-2023 (2012). Zhu, R. \& Buchwald, S. L. Enantioselective Functionalization of Radical Intermediates in Redox Catalysis: Copper-Catalyzed Asymmetric Oxytrifluoromethylation of Alkenes. Angew. Chem. Int. Ed. 52, 12655-12658 (2013).

27 Kainz, Q. M. et al. Asymmetric copper-catalyzed C-N cross-couplings induced by visible light. Science 351, 681684 (2016).

28 Zhang, W. et al. Enantioselective cyanation of benzylic C-H bonds via copper-catalyzed radical relay. Science 353, 1014-1018 (2016).

29 Gutierrez, O., Tellis, J. C., Primer, D. N., Molander, G. A. \& Kozlowski, M. C. Nickel-Catalyzed Cross-Coupling of Photoredox-Generated Radicals: Uncovering a General Manifold for Stereoconvergence in Nickel-Catalyzed Cross-Couplings. J. Am. Chem. Soc. 137, 4896-4899 (2015).

30 Zuo, Z. et al. Enantioselective Decarboxylative Arylation of $\alpha$-Amino Acids via the Merger of Photoredox and Nickel Catalysis. J. Am. Chem. Soc. 138, 1832-1835 (2016).

31 Wang, Z., Yin, H. \& Fu, G. C. Catalytic enantioconvergent coupling of secondary and tertiary electrophiles with olefins. Nature 563, 379-383 (2018).

32 Zhao, Y. \& Weix, D. J. Enantioselective Cross-Coupling of meso-Epoxides with Aryl Halides. J. Am. Chem. Soc. 137, 3237-3240 (2015).

33 Hao, W., Harenberg, J. H., Wu, X., MacMillan, S. N. \& Lin, S. Diastereo- and Enantioselective Formal [3 + 2] Cycloaddition of Cyclopropyl Ketones and Alkenes via Ti-Catalyzed Radical Redox Relay. J. Am. Chem. Soc. 140, 3514-3517 (2018).

34 Wang, Y., Wen, X., Cui, X. \& Zhang, X. P. Enantioselective Radical Cyclization for Construction of 5Membered Ring Structures by Metalloradical C-H Alkylation. J. Am. Chem. Soc. 140, 4792-4796 (2018).

35 Lang, K., Torker, S., Wojtas, L. \& Zhang, X. P. Asymmetric Induction and Enantiodivergence in Catalytic Radical C-H Amination via Enantiodifferentiative H-Atom Abstraction and Stereoretentive Radical Substitution. J. Am. Chem. Soc. 141, 12388-12396 (2019).

36 Knowles, R. R. \& Jacobsen, E. N. Attractive noncovalent interactions in asymmetric catalysis: Links between enzymes and small molecule catalysts. Proc. Natl. Acad. Sci. USA 107, 20678-20685 (2010).

37 Dalko, P. I. Comprehensive Enantioselective Organocatalysis: Catalysts, Reactions, and Applications

(Wiley-VCH, Weinheim, 2013).

38 Doyle, A. G. \& Jacobsen, E. N. Small-Molecule H-Bond Donors in Asymmetric Catalysis. Chem. Rev. 107, 57135743 (2007).

39 Phipps, R. J., Hamilton, G. L. \& Toste, F. D. The progression of chiral anions from concepts to applications in asymmetric catalysis. Nature Chemistry 4, 603 (2012).

40 Brak, K. \& Jacobsen, E. N. Asymmetric Ion-Pairing Catalysis. Angew. Chem. Int. Ed. 52, 534-561 (2013).

41 Mahlau, M. \& List, B. Asymmetric Counteranion-Directed Catalysis: Concept, Definition, and Applications. Angew. Chem. Int. Ed. 52, 518-533 (2013).

42 Parmar, D., Sugiono, E., Raja, S. \& Rueping, M. Complete Field Guide to Asymmetric BINOL-Phosphate Derived Brønsted Acid and Metal Catalysis: History and Classification by Mode of Activation; Brønsted Acidity, Hydrogen Bonding, Ion Pairing, and Metal Phosphates. Chem. Rev. 114, 9047-9153 (2014).

43 Akiyama, T., Itoh, J., Yokota, K. \& Fuchibe, K. Enantioselective Mannich-Type Reaction Catalyzed by a Chiral Brønsted Acid. Angew. Chem. Int. Ed. 43, 1566-1568 (2004).

44 Uraguchi, D. \& Terada, M. Chiral Brønsted Acid-Catalyzed Direct Mannich Reactions via Electrophilic Activation. J. Am. Chem. Soc. 126, 5356-5357 (2004).

45 Reid, J. P., Simón, L. \& Goodman, J. M. A Practical Guide for Predicting the Stereochemistry of Bifunctional Phosphoric Acid Catalyzed Reactions of Imines. Acc. Chem. Res. 49, 1029-1041 (2016).

46 Kennedy, C. R., Lin, S. \& Jacobsen, E. N. The Cation- $\pi$ Interaction in Small-Molecule Catalysis. Angew. Chem. Int. Ed. 55, 12596-12624 (2016).

47 Zhao, Y. et al. The Emergence of Anion- $\pi$ Catalysis. Acc. Chem. Res. 51, 2255-2263 (2018). 
Sutar, R. L. \& Huber, S. M. Catalysis of Organic Reactions through Halogen Bonding. ACS Catal. 9, 9622-9639 (2019).

49 Wagner, J. P. \& Schreiner, P. R. London Dispersion in Molecular Chemistry-Reconsidering Steric Effects. Angew. Chem. Int. Ed. 54, 12274-12296 (2015).

50 Neel, A. J., Hilton, M. J., Sigman, M. S. \& Toste, F. D. Exploiting non-covalent $\pi$ interactions for catalyst design. Nature 543, 637-646 (2017).

51 Wheeler, S. E., Seguin, T. J., Guan, Y. \& Doney, A. C. Noncovalent Interactions in Organocatalysis and the Prospect of Computational Catalyst Design. Acc. Chem. Res. 49, 1061-1069 (2016). Ahn, S., Hong, M., Sundararajan, M., Ess, D. H. \& Baik, M.-H. Design and Optimization of Catalysts Based on Mechanistic Insights Derived from Quantum Chemical Reaction Modeling. Chem. Rev. 119, 6509-6560 (2019). Brimioulle, R., Lenhart, D., Maturi, M. M. \& Bach, T. Enantioselective Catalysis of Photochemical Reactions. Angew. Chem. Int. Ed. 54, 3872-3890 (2015).

54 Jiang, C., Chen, W., Zheng, W.-H. \& Lu, H. Advances in asymmetric visible-light photocatalysis, 2015-2019. Org. Biomol. Chem. 17, 8673-8689 (2019). Yin, Y., Zhao, X., Qiao, B. \& Jiang, Z. Cooperative photoredox and chiral hydrogen-bonding catalysis. Org. Chem. Front. 7, 1283-1296 (2020). Gamlin, J. N. et al. The Ionic Auxiliary Concept in Solid State Organic Photochemistry. Acc. Chem. Res. 29, 203209 (1996).

57 Jones, R., Scheffer, J. R., Trotter, J. \& Yang, J. Crystal to molecular chirality transfer: Supramolecular photochemistry of crystalline carboxylate salts. Tetrahedron Lett. 33, 5481-5484 (1992). Burg, F. \& Bach, T. Lactam Hydrogen Bonds as Control Elements in Enantioselective Transition-MetalCatalyzed and Photochemical Reactions. J. Org. Chem. 84, 8815-8836 (2019). 2-Quinolone in Solution. J. Am. Chem. Soc. 122, 11525-11526 (2000). Bach, T., Bergmann, H., Grosch, B. \& Harms, K. Highly Enantioselective Intra- and Intermolecular [2 + 2] Photocycloaddition Reactions of 2-Quinolones Mediated by a Chiral Lactam Host: Host-Guest Interactions, Product Configuration, and the Origin of the Stereoselectivity in Solution. J. Am. Chem. Soc. 124, 7982-7990 (2002).

61 Aechtner, T., Dressel, M. \& Bach, T. Hydrogen Bond Mediated Enantioselectivity of Radical Reactions. Angew. Chem. Int. Ed. 43, 5849-5851 (2004).

62 Dressel, M. \& Bach, T. Chirality Multiplication and Efficient Chirality Transfer in exo- and endo-Radical Cyclization Reactions of 4-(4‘-Iodobutyl)quinolones. Org. Lett. 8, 3145-3147 (2006). Bakowski, A., Dressel, M., Bauer, A. \& Bach, T. Enantioselective radical cyclisation quinolones mediated by a chiral template. Org. Biomol. Chem. 9, 3516-3529 (2011). to 3-Alkylidene Indolin-2-ones. Chem. Eur J. 22, 6519-6523 (2016). electron transfer. Nature 436, 1139-1140 (2005).

67 Müller, C. et al. Enantioselective Intramolecular [2 + 2]-Photocycloaddition Reactions of 4-Substituted Quinolones Catalyzed by a Chiral Sensitizer with a Hydrogen-Bonding Motif. J. Am. Chem. Soc. 133, 1668916697 (2011).

68 Alonso, R. \& Bach, T. A Chiral Thioxanthone as an Organocatalyst for Enantioselective [2+2] Photocycloaddition Reactions Induced by Visible Light. Angew. Chem. Int. Ed. 53, 4368-4371 (2014). Tröster, A., Alonso, R., Bauer, A. \& Bach, T. Enantioselective Intermolecular [2 + 2] Photocycloaddition Reactions of 2(1H)-Quinolones Induced by Visible Light Irradiation. J. Am. Chem. Soc. 138, 7808-7811 (2016). Hölzl-Hobmeier, A. et al. Catalytic deracemization of chiral allenes by sensitized excitation with visible light. Nature 564, 240-243 (2018).

71 Tröster, A., Bauer, A., Jandl, C. \& Bach, T. Enantioselective Visible-Light-Mediated Formation of 3Cyclopropylquinolones by Triplet-Sensitized Deracemization. Angew. Chem. Int. Ed. 58, 3538-3541 (2019). Bergonzini, G., Schindler, C. S., Wallentin, C.-J., Jacobsen, E. N. \& Stephenson, C. R. J. Photoredox activation and anion binding catalysis in the dual catalytic enantioselective synthesis of $\beta$-amino esters. Chem. Sci. 5, 112116 (2014).

73 Vallavoju, N., Selvakumar, S., Jockusch, S., Sibi, M. P. \& Sivaguru, J. Enantioselective Organo-Photocatalysis Mediated by Atropisomeric Thiourea Derivatives. Angew. Chem. Int. Ed. 53, 5604-5608 (2014). 
Chen, L.-A. et al. Asymmetric Catalysis with an Inert Chiral-at-Metal Iridium Complex. J. Am. Chem. Soc. 135, 10598-10601 (2013).

75 Skubi, K. L. et al. Enantioselective Excited-State Photoreactions Controlled by a Chiral Hydrogen-Bonding Iridium Sensitizer. J. Am. Chem. Soc. 139, 17186-17192 (2017).

76 Zheng, J. et al. Enantioselective Intermolecular Excited-State Photoreactions Using a Chiral Ir Triplet Sensitizer: Separating Association from Energy Transfer in Asymmetric Photocatalysis. J. Am. Chem. Soc. 141, 1362513634 (2019).

77 Uraguchi, D., Kinoshita, N., Kizu, T. \& Ooi, T. Synergistic Catalysis of Ionic Brønsted Acid and Photosensitizer for a Redox Neutral Asymmetric $\alpha$-Coupling of N-Arylaminomethanes with Aldimines. J. Am. Chem. Soc. 137, 13768-13771 (2015).

78 Kizu, T., Uraguchi, D. \& Ooi, T. Independence from the Sequence of Single-Electron Transfer of Photoredox Process in Redox-Neutral Asymmetric Bond-Forming Reaction. J. Org. Chem. 81, 6953-6958 (2016).

79 Shirakawa, S. \& Maruoka, K. Recent Developments in Asymmetric Phase-Transfer Reactions. Angew. Chem. Int. Ed. 52, 4312-4348 (2013).

80 Cannizzaro, C. E. \& Houk, K. N. Magnitudes and Chemical Consequences of R3N+-C-H $\cdots$ OC Hydrogen Bonding. J. Am. Chem. Soc. 124, 7163-7169 (2002).

81 Woźniak, Ł., Murphy, J. J. \& Melchiorre, P. Photo-organocatalytic Enantioselective Perfluoroalkylation of $\beta$ Ketoesters. J. Am. Chem. Soc. 137, 5678-5681 (2015).

82 Yang, C. et al. Origin of Stereoselectivity of the Photoinduced Asymmetric Phase-Transfer-Catalyzed Perfluoroalkylation of $\beta$-Ketoesters. J. Org. Chem. 82, 9321-9327 (2017).

83 Lin, L. et al. Organocatalytic Enantioselective Protonation for Photoreduction of Activated Ketones and Ketimines Induced by Visible Light. Angew. Chem. Int. Ed. 56, 13842-13846 (2017).

84 Hou, M. et al. Enantioselective photoredox dehalogenative protonation. Chem. Sci. 10, 6629-6634 (2019).

85 Lee, S. \& Kim, S. Enantioselective radical addition reactions to imines using binaphthol-derived chiral N-triflyl phosphoramides. Tetrahedron Lett. 50, 3345-3348 (2009).

86 Rono, L. J., Yayla, H. G., Wang, D. Y., Armstrong, M. F. \& Knowles, R. R. Enantioselective Photoredox Catalysis Enabled by Proton-Coupled Electron Transfer: Development of an Asymmetric Aza-Pinacol Cyclization. J. Am. Chem. Soc. 135, 17735-17738 (2013).

87 Proctor, R. S. J., Davis, H. J. \& Phipps, R. J. Catalytic enantioselective Minisci-type addition to heteroarenes. Science 360, 419-422 (2018).

88 Sigman, M. S., Harper, K. C., Bess, E. N. \& Milo, A. The Development of Multidimensional Analysis Tools for Asymmetric Catalysis and Beyond. Acc. Chem. Res. 49, 1292-1301 (2016).

89 Reid, J. P., Proctor, R. S. J., Sigman, M. S. \& Phipps, R. J. Predictive Multivariate Linear Regression Analysis Guides Successful Catalytic Enantioselective Minisci Reactions of Diazines. J. Am. Chem. Soc. 141, 19178-19185 (2019).

90 Liu, X. et al. Organocatalytic Enantioselective Addition of $\alpha$-Aminoalkyl Radicals to Isoquinolines. Org. Lett. 20, 6298-6301 (2018).

91 Zheng, D. \& Studer, A. Asymmetric Synthesis of Heterocyclic $\gamma$-Amino-Acid and Diamine Derivatives by ThreeComponent Radical Cascade Reactions. Angew. Chem. Int. Ed. 58, 15803-15807 (2019).

92 Liu, Y. et al. Catalytic enantioselective radical coupling of activated ketones with N-aryl glycines. Chem. Sci. 9, 8094-8098 (2018).

$93 \mathrm{Li}$, J. et al. Formal enantioconvergent substitution of alkyl halides via catalytic asymmetric photoredox radical coupling. Nat. Commun. 9, 2445 (2018).

94 Zeng, G., Li, Y., Qiao, B., Zhao, X. \& Jiang, Z. Photoredox asymmetric catalytic enantioconvergent substitution of 3-chlorooxindoles. Chem. Commun. 55, 11362-11365 (2019).

95 Yin, Y. et al. Conjugate Addition-Enantioselective Protonation of N-Aryl Glycines to $\alpha$-Branched 2Vinylazaarenes via Cooperative Photoredox and Asymmetric Catalysis. J. Am. Chem. Soc. 140, 6083-6087 (2018).

96 Cao, K. et al. Catalytic Enantioselective Addition of Prochiral Radicals to Vinylpyridines. J. Am. Chem. Soc. 141, 5437-5443 (2019).

97 Morse, P. D., Nguyen, T. M., Cruz, C. L. \& Nicewicz, D. A. Enantioselective counter-anions in photoredox catalysis: The asymmetric cation radical Diels-Alder reaction. Tetrahedron 74, 3266-3272 (2018).

98 Gentry, E. C., Rono, L. J., Hale, M. E., Matsuura, R. \& Knowles, R. R. Enantioselective Synthesis of Pyrroloindolines via Noncovalent Stabilization of Indole Radical Cations and Applications to the Synthesis of Alkaloid Natural Products. J. Am. Chem. Soc. 140, 3394-3402 (2018). 

excited state electron transfer. Science 366, 364-369 (2019).

100 Roos, C. B., Demaerel, J., Graff, D. E. \& Knowles, R. R. Enantioselective Hydroamination of Alkenes with Sulfonamides Enabled by Proton-Coupled Electron Transfer. J. Am. Chem. Soc. 142, 5974-5979 (2020).

101 Huang, X. \& Groves, J. T. Beyond ferryl-mediated hydroxylation: 40 years of the rebound mechanism and C-H activation. J. Biol. Inorg. Chem. 22, 185-207 (2017).

102 Huang, X. \& Groves, J. T. Oxygen Activation and Radical Transformations in Heme Proteins and Metalloporphyrins. Chem. Rev. 118, 2491-2553 (2018).

103 Frost, J. R., Huber, S. M., Breitenlechner, S., Bannwarth, C. \& Bach, T. Enantiotopos-Selective C-H Oxygenation Catalyzed by a Supramolecular Ruthenium Complex. Angew. Chem. Int. Ed. 54, 691-695 (2015).

104 Burg, F., Gicquel, M., Breitenlechner, S., Pöthig, A. \& Bach, T. Site- and Enantioselective C-H Oxygenation Catalyzed by a Chiral Manganese Porphyrin Complex with a Remote Binding Site. Angew. Chem. Int. Ed. 57, 2953-2957 (2018).

105 Burg, F., Breitenlechner, S., Jandl, C. \& Bach, T. Enantioselective oxygenation of exocyclic methylene groups by a manganese porphyrin catalyst with a chiral recognition site. Chem. Sci. 11, 2121-2129 (2020).

106 Gu, Q.-S., Li, Z.-L. \& Liu, X.-Y. Copper(I)-Catalyzed Asymmetric Reactions Involving Radicals. Acc. Chem. Res. 53, 170-181 (2020).

107 Lin, J.-S. et al. A Dual-Catalytic Strategy To Direct Asymmetric Radical Aminotrifluoromethylation of Alkenes. J. Am. Chem. Soc. 138, 9357-9360 (2016).

108 Wang, F.-L. et al. Catalytic Asymmetric Radical Diamination of Alkenes. Chem 3, 979-990 (2017).

109 Ye, L., Tian, Y., Meng, X., Gu, Q.-S. \& Liu, X.-Y. Enantioselective Copper(I)/Chiral Phosphoric Acid Catalyzed Intramolecular Amination of Allylic and Benzylic C-H Bonds. Angew. Chem. Int. Ed. 59, 1129-1133 (2020).

110 Cheng, Y. F. et al. Catalytic enantioselective desymmetrizing functionalization of alkyl radicals via $\mathrm{Cu}(\mathrm{i}) / \mathrm{CPA}$ cooperative catalysis. Nat. Catal. 3, 401-410 (2020).

111 Hönig, M., Sondermann, P., Turner, N. J. \& Carreira, E. M. Enantioselective Chemo- and Biocatalysis: Partners in Retrosynthesis. Angew. Chem. Int. Ed. 56, 8942-8973 (2017).

112 Sheldon, R. A., Brady, D. \& Bode, M. L. The Hitchhiker's guide to biocatalysis: recent advances in the use of enzymes in organic synthesis. Chem. Sci. 11, 2587-2605 (2020).

113 Reetz, M. T. Biocatalysis in Organic Chemistry and Biotechnology: Past, Present, and Future. J. Am. Chem. Soc. 135, 12480-12496 (2013).

114 Arnold, F. H. Directed Evolution: Bringing New Chemistry to Life. Angew. Chem. Int. Ed. 57, 4143-4148 (2018).

115 Leveson-Gower, R. B., Mayer, C. \& Roelfes, G. The importance of catalytic promiscuity for enzyme design and evolution. Nat. Rev. Chem. 3, 687-705 (2019).

116 Chen, K. \& Arnold, F. H. Engineering new catalytic activities in enzymes. Nat. Catal. 3, 203-213 (2020).

117 Sandoval, B. A. \& Hyster, T. K. Emerging strategies for expanding the toolbox of enzymes in biocatalysis. Curr. Opin. Chem. Biol. 55, 45-51 (2020).

118 Broderick, J. B., Duffus, B. R., Duschene, K. S. \& Shepard, E. M. Radical S-Adenosylmethionine Enzymes. Chem. Rev. 114, 4229-4317 (2014).

119 Shibata, N. \& Toraya, T. Molecular architectures and functions of radical enzymes and their (re)activating proteins. The Journal of Biochemistry 158, 271-292 (2015).

120 Jäger, C. M. \& Croft, A. K. Anaerobic Radical Enzymes for Biotechnology. ChemBioEng Rev. 5, 143-162 (2018).

121 Emmanuel, M. A., Greenberg, N. R., Oblinsky, D. G. \& Hyster, T. K. Accessing non-natural reactivity by irradiating nicotinamide-dependent enzymes with light. Nature 540, 414-417 (2016).

122 Fukuzumi, S., Hironaka, K. \& Tanaka, T. Photoreduction of alkyl halides by an NADH model compound. An electron-transfer chain mechanism. J. Am. Chem. Soc. 105, $4722-4727$ (1983).

123 Biegasiewicz, K. F., Cooper, S. J., Emmanuel, M. A., Miller, D. C. \& Hyster, T. K. Catalytic promiscuity enabled by photoredox catalysis in nicotinamide-dependent oxidoreductases. Nat. Chem. 10, 770-775 (2018).

124 Sandoval, B. A., Kurtoic, S. I., Chung, M. M., Biegasiewicz, K. F. \& Hyster, T. K. Photoenzymatic Catalysis Enables Radical-Mediated Ketone Reduction in Ene-Reductases. Angew. Chem. Int. Ed. 58, 8714-8718 (2019). Nakano, Y. et al. Photoenzymatic Hydrogenation of Heteroaromatic Olefins Using 'Ene'-Reductases with Photoredox Catalysts. Angew. Chem. Int. Ed. 59, 10484-10488 (2020).

126 Sandoval, B. A., Meichan, A. J. \& Hyster, T. K. Enantioselective Hydrogen Atom Transfer: Discovery of Catalytic Promiscuity in Flavin-Dependent 'Ene’-Reductases. J. Am. Chem. Soc. 139, 11313-11316 (2017).

127 Biegasiewicz, K. F. et al. Photoexcitation of flavoenzymes enables a stereoselective radical cyclization. Science 364, 1166-1169 (2019). 
128 Black, M. J. et al. Asymmetric redox-neutral radical cyclization catalysed by flavin-dependent 'ene'-reductases. Nat. Chem. 12, 71-75 (2020).

\section{Table of contents summary:}

The past decade has seen unprecedented growth in the development of new chemical methods that proceed by mechanisms involving radical intermediates, but controlling absolute stereochemistry has been a long-standing challenge. This Review Article will examine developments that utilise attractive non-covalent interactions between a chiral catalyst and the substrate to exert enantiocontrol in radical reactions.

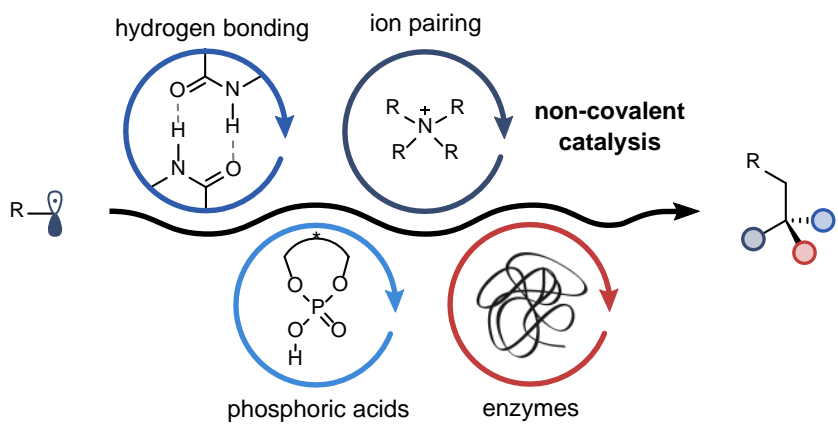




\section{Figure legends:}

$a$

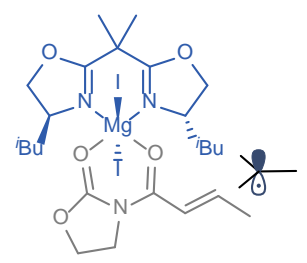

Lewis acid catalysis

(Sibi, Porter and co-workers, 1996) $^{12}$

C

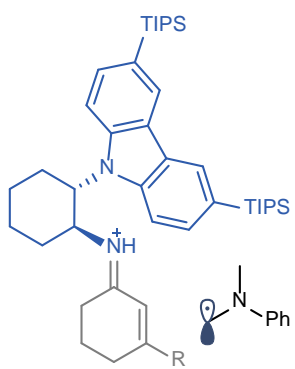

iminium catalysis

(Melchiorre and co-workers, 2016) $^{18}$

e

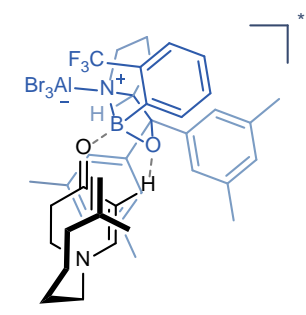

Lewis acid-catalysed photocycloaddition (Brimioulle and Bach, 2013) $^{20}$ b

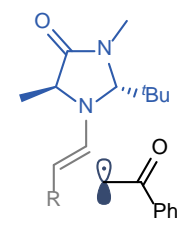

enamine catalysis

$\left(_{\text {Nicewicz and MacMillan, 2008) }}^{16}\right.$

d

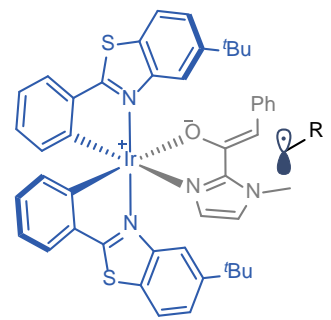

dual Lewis acid / photoredox catalyst (Meggers and co-workers, 2014) ${ }^{19}$

f

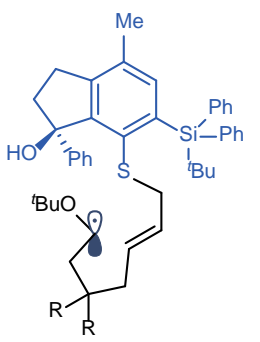

thiyl radical catalysis (Maruoka and co-workers, 2014) $^{22}$

h

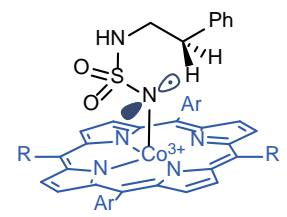

metalloradical-based functionalisation

(Zhang and co-workers, 2019) $^{35}$

copper-catalysed radical relay (Stahl, Liu and co-workers, 2016) 28

Figure 1. A selection of enantioselective radical reactions to date, enabled by primarily covalent modes of catalysis. a, Chiral Lewis acid catalysis facilitates control over the enantioselectivity of radical addition to an oxazolidinone-derived Michael-type acceptor. b, An imidazolidinone organocatalyst undergoes in-situ enamine formation, providing the required enantiofacial discrimination for selective radical addition to the enamine. c, Organocatalytic substrate activation via iminium-ion formation facilitates enantioselective radical conjugate addition to cyclic $\beta$ substituted enones. d, Tandem Lewis acid/photoredox catalytic system - a chiral iridium complex acts as a photoredox catalyst and exerts control over asymmetry in enantioselective radical addition to 2-acyl imidazoles. e, Catalytic enantioselective photocycloaddition achieved by selective photoexcitation of a chiral Lewis-acid-complexed enone. $\mathbf{f}$, A chiral organic thiyl radical catalyst enables highly enantioselective radical cyclisations. $\mathbf{g}$, Addition of prochiral radicals to chiral copper complexes allows enantioselective benzylic cyanation. $\mathbf{h}$, Asymmetric hydrogen atom abstraction and stereoretentive radical amination by chiral cobalt-porphyrin complexes. 


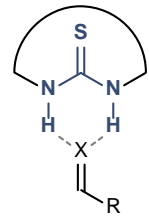

hydrogen bond

donor

d

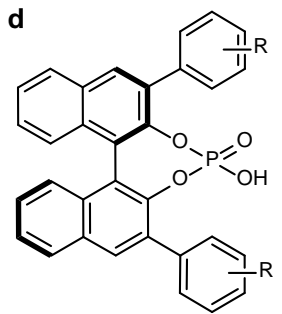

e

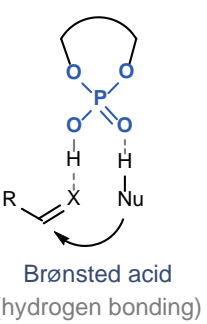

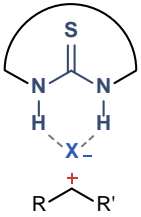

anionhpinding

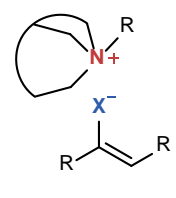

ion pairing

(chiral cation)

Generic structure of a 3,3'-diaryl substituted BINOL-phosphoric acid

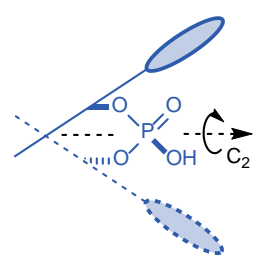

f

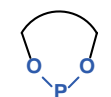

O

$\mathrm{x}^{+} \overbrace{\mathrm{Nu}}^{\mathrm{H}}$

chiral anion (in organocatalysis) g

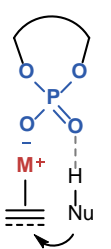

chiral anion

(with transition
metals)

Figure 2 Summary of commonly used modes in non-covalent enantioselective catalysis. a, Hydrogen bond donor catalysis: chiral thioureacontaining scaffolds activate neutral basic substrates towards addition of nucleophiles. $\mathbf{b}$, Anion-binding catalysis: activation of substrate via thioureaassisted binding to conjugate anion of a prochiral cationic intermediate. c, Archetypal ion-pairing interaction between anionic intermediate and chiral cationic catalyst, as encountered in asymmetric phase-transfer catalysis. d, Overview of structure of BINOL-derived phosphoric acid (left) with alternative representation (right) highlighting the C2-symmetric pocket containing the phosphoric acid functionality. e, Phosphoric acids as bifunctional Brønsted acid catalysts with activation of basic functionality in one substrate via the acidic proton while the Lewis basic oxygen engages a second substrate via hydrogen bonding. f, Phosphates as chiral anions in organocatalysis engage a cationic intermediate via ion-pairing. $\mathbf{g}$, Phosphates as chiral anions in combination with transition metal catalysis. In both $\mathbf{f}$ and $\mathbf{g}$ the phosphate retains its dual functionality, able to engage the nucleophile via hydrogen bonding with the Lewis basic oxygen of the phosphate. 


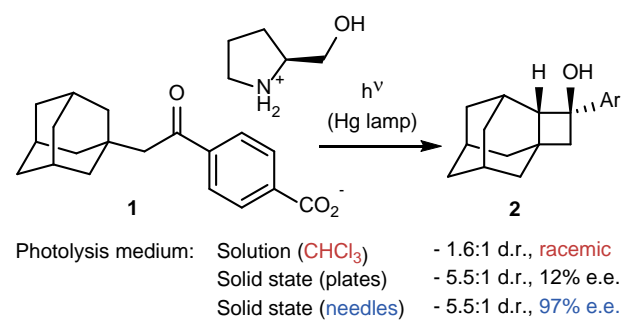

c<smiles></smiles>
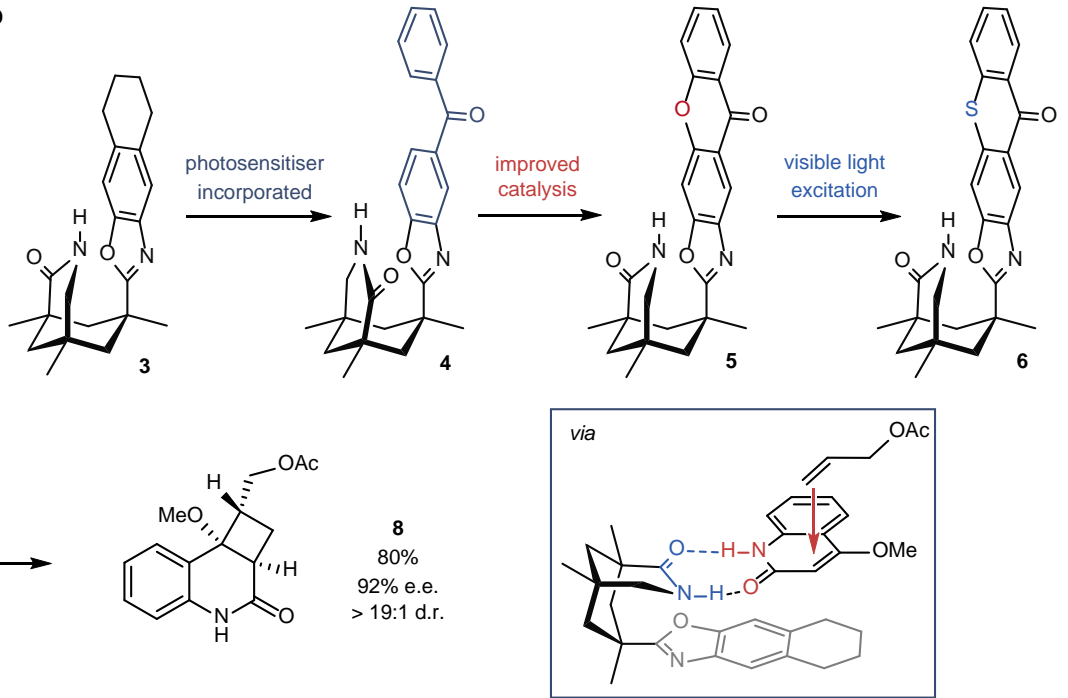

d<smiles>O=c1cc(CCN2CCCC2)c2ccccc2[nH]1</smiles>

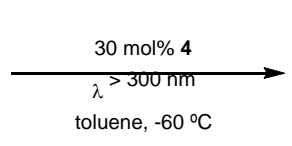<smiles>O=C1C[C@]2(CCN3CCC[C@H]32)c2ccccc2N1</smiles>

10
$64 \%$ $70 \%$ e.e.
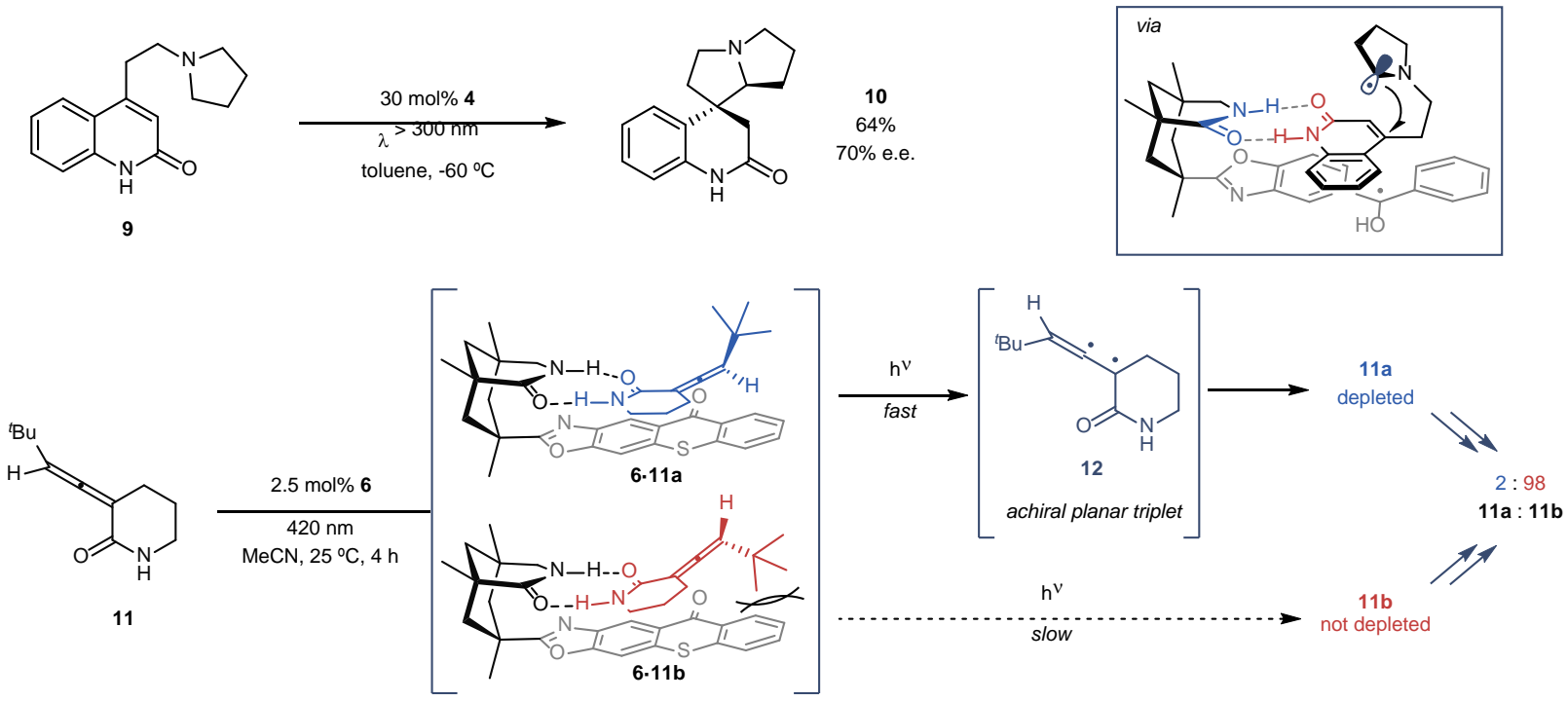<smiles>C=CCCCc1cc(=O)oc2cc(F)ccc12</smiles>

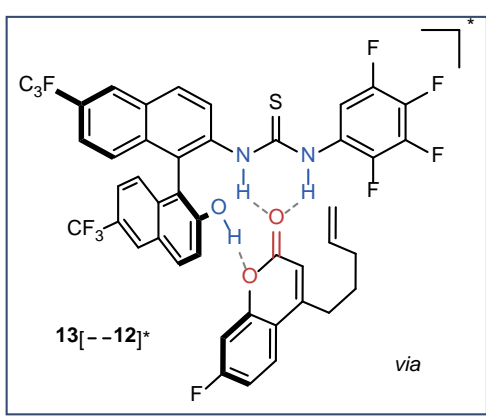<smiles>O=C1Oc2cc(F)ccc2[C@@]23CCC[C@H]2C[C@H]13</smiles>

14

$100 \%$ conversion

$90 \%$ e.e.<smiles>C=CCCOc1cc2cc([13F])ccc2[nH]c1=O</smiles>

15
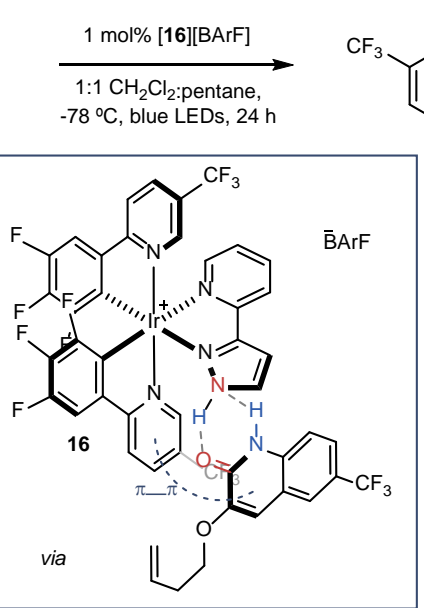<smiles>O=C1Nc2ccc(C(F)(F)F)cc2[C@H]2CCCC3CCC1C32</smiles>

17

$97 \%$ $84 \%$ e.e.

Figure 3 Design approach and selected applications of chiral hydrogen bonding templates and photosensitisers. a, Asymmetric Norrish type II cyclisation of prolinol salts of adamantyl acetophenones such as $\mathbf{1}$, with high enantioselectivity obtained in the crystalline needle form. $\mathbf{b}$, The evolution of chiral lactam hosts from original template $\mathbf{3}$ through incorporating a photosensitive benzophenone unit, $\mathbf{4}$, a rigid xanthone unit, $\mathbf{5}$, and a visible-light absorbing thioxanthone unit, 6. c, Asymmetric [2+2] photocycloaddition between alkoxyquinolone 7 and unactivated alkenes controlled by directional hydrogen bonding to template 3 , which blocks one face of the substrate. $\mathbf{d}$, Asymmetric cyclisation of an $\alpha$-aminoalkyl radical generated on the pyrrolidine unit of $\mathbf{9}$ through photochemical activation by sensitiser $\mathbf{4}$. e, Catalytic deracemization of allenes. Enantiomer 11a is gradually depleted by more efficient energy transfer from, and binding to, $\mathbf{6}$, which racemises the substrate via planar biradical $\mathbf{1 2}$, leaving $\mathbf{1 1 b}$ enriched. $\mathbf{f}$, Enantioselective [2+2] cyclisation of coumarins mediated by hydrogen bonding interactions with chiral thiourea $\mathbf{1 3}$. $\mathbf{g}$, chiral iridium sensitiser $\mathbf{1 6}$ induces asymmetry in [2+2] cycloadditions. Multiple contacts between the lactam and $\mathrm{N}-\mathrm{H}$ of the substrate $\mathbf{1 5}$ are proposed to form a highly organised environment for cyclisation, along with $\pi-\pi$ stacking interactions between the lactam ring and the pyridyl unit of the ppy ligand of $\mathbf{1 6}$. 
a
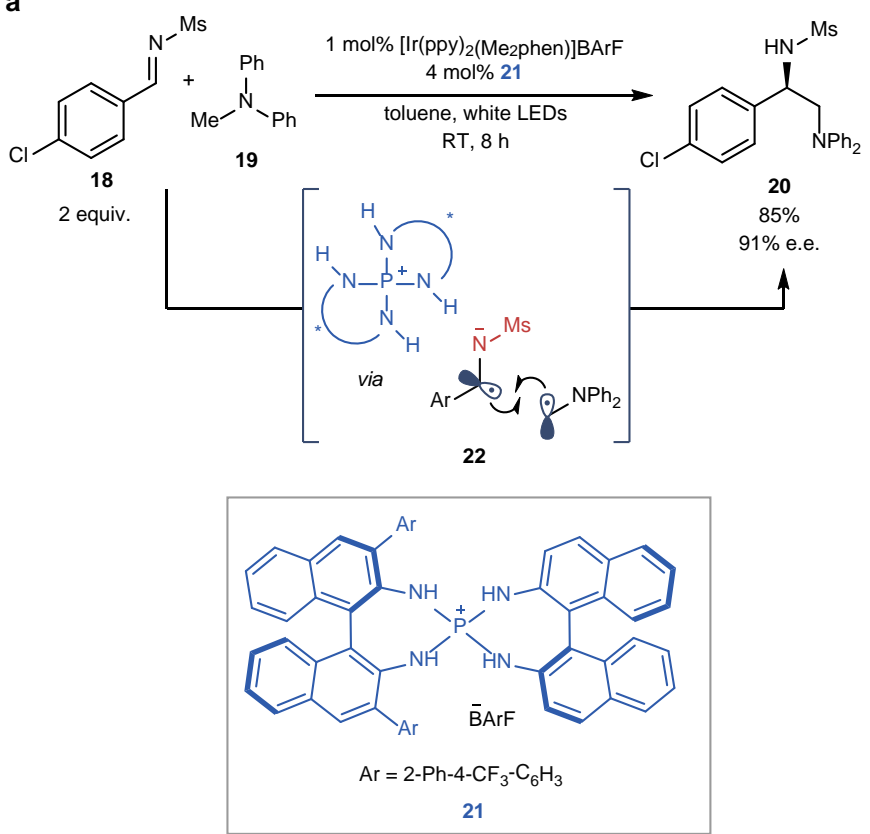

b
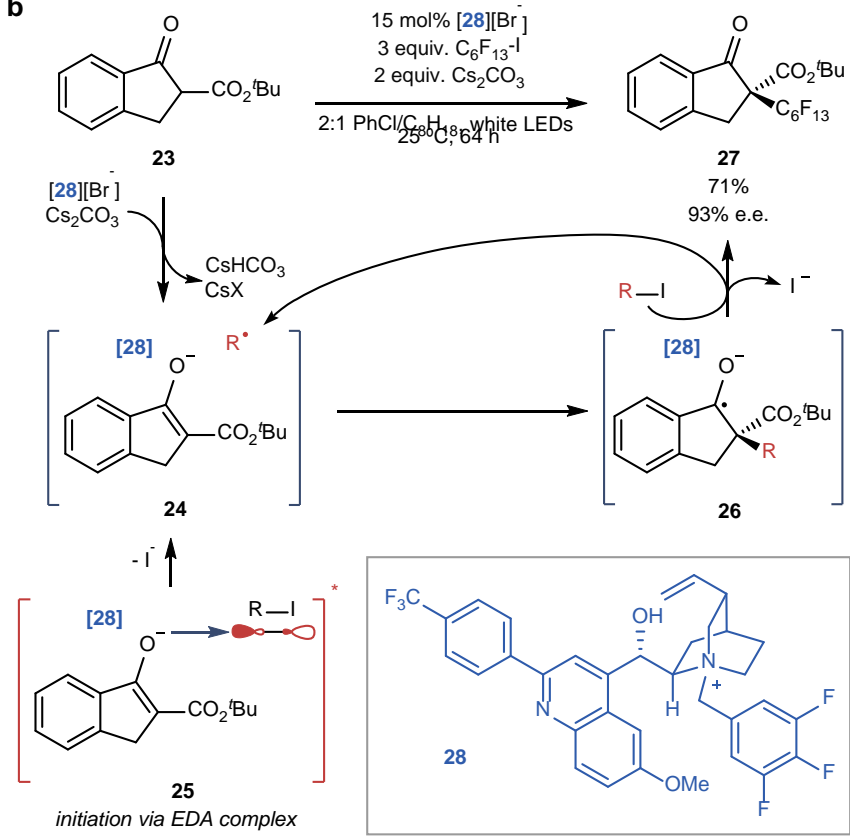<smiles>O=C(C(=O)c1ccccc1)c1ccccc1</smiles>

29

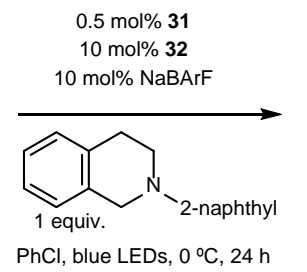

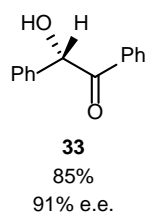
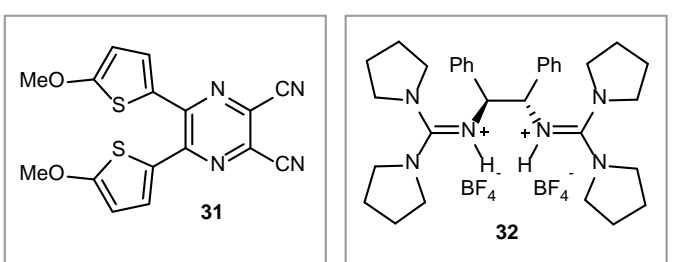

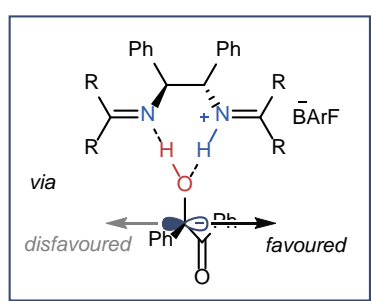

Figure 4 Cationic hydrogen bond donors in asymmetric photoredox-mediated processes. a, A radical-radical coupling is rendered enantioselective by chiral phosphonium catalyst $\mathbf{2 1}$, which ion pairs to an imine-derived radical anion (22). $\mathbf{b}$, Cinchonine-derived ammonium cation $\mathbf{2 8}$ induces asymmetry in perfluoroalkylation of $\beta$-ketoesters. The reaction is initiated through an electron-donor-acceptor complex (25) between the key enolate intermediate and the alkyl iodide precursor and proceeds via a chain process. Multiple hydrogen bonds between the enolate and chiral cation allow high levels of enantioenrichment. c, Asymmetric protonation of $\alpha$-oxyanions, generated through reduction of dicarbonyls such as $\mathbf{2 9}$, is realised by cation 32. The $C_{2}$ symmetry allows blocking of one face of these photoreductively generated species, resulting in enantioselective protonation. 
a

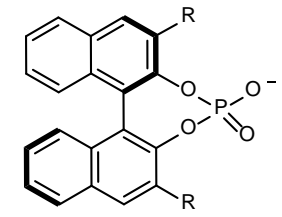

(R)-34: $\mathrm{R}=\mathrm{SiPh}_{3}$

(R)-35: $\mathrm{R}=2,4,6-\left({ }^{i} \mathrm{Pr}\right)_{3} \mathrm{C}_{6} \mathrm{H}_{2}$ (S)-36: $\mathrm{R}=9$-pyrenyl

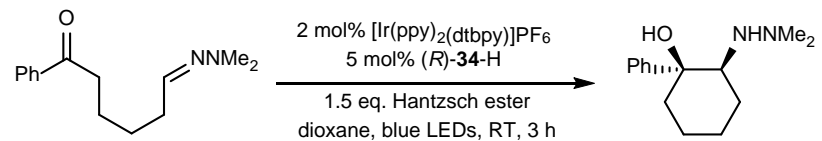

42

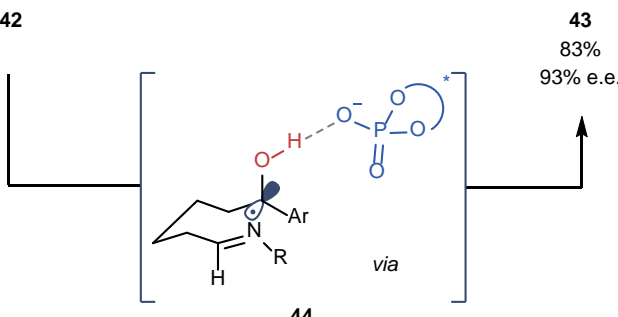

44 d

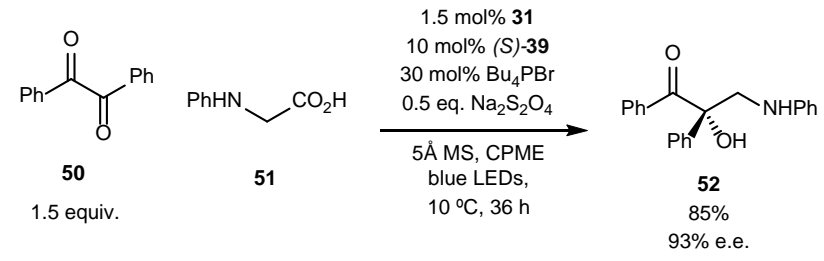<smiles>CCCCCCCCCC(=O)C(=O)c1ccccc1</smiles>

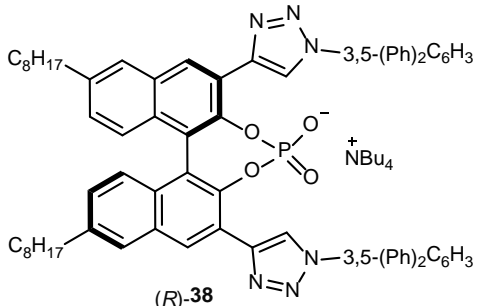

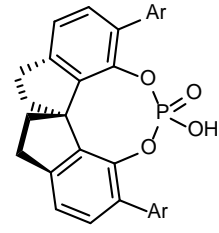

(S)-39: $\mathrm{Ar}=2,6-\mathrm{Me}_{2}-4-{ }^{\mathrm{t}} \mathrm{Bu}$ (S) $-40 \mathrm{Ar}=2,4,6-\left({ }^{i} \mathrm{Pr}\right)_{3} \mathrm{C}_{6} \mathrm{H}_{2}$ (S)-41: $\mathrm{Ar}=4-\mathrm{Ph}-\mathrm{C}_{6} \mathrm{H}_{4}$
$1 \mathrm{~mol} \%$

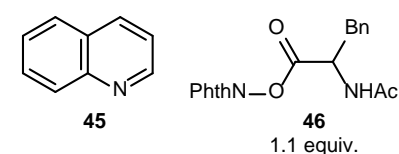

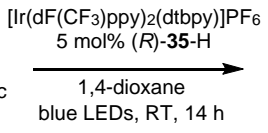

blue LEDs, RT, $14 \mathrm{~h}$

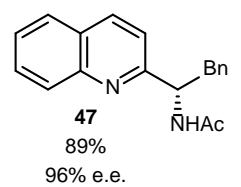

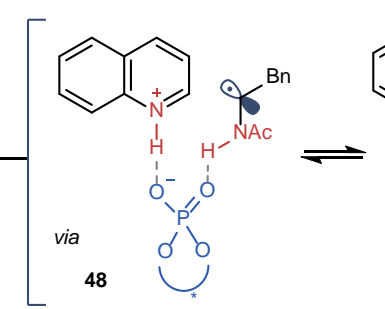

e<smiles>C=Cc1ccccn1</smiles><smiles>CC(=O)c1ccccc1</smiles>

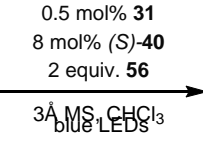

$-5^{\circ} \mathrm{C}, 17 \mathrm{~h}$

1.5 equiv

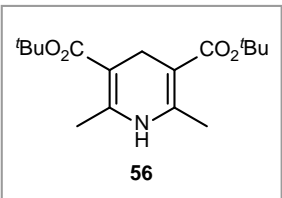

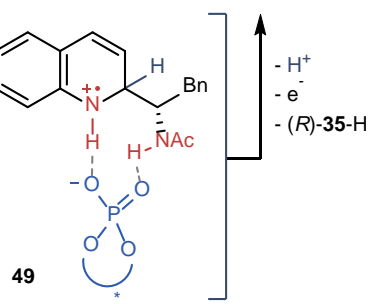

\section{Ac}

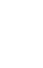

Figure 5 Chiral phosphoric acids as enantioselective catalysts in radical-based reactions. a, Selected examples of chiral phosphoric acids employed in asymmetric radical reactions discussed herein. $\mathbf{b}$, Enantioselective aza-pinacol coupling via acid-promoted PCET. The chiral phosphate $\mathbf{3 4}$ remains hydrogen-bonded to the neutral ketyl radical during enantiodetermining cyclisation (44). c, Asymmetric Minisci-type reaction of $\alpha$-amido-alkyl radicals. Enantioselectivity is thought to be determined by selective deprotonation of one diastereomeric intermediate after reversible radical addition (48 to 49). d, Proposed radical-radical coupling between ketyl radicals and $\alpha$-amino radicals mediated by a chiral phosphoric acid. e, Enantioselective addition of neutral ketyl radicals to vinylpyridines such as $\mathbf{5 4}$ allows the construction of remote stereocentres in a net reductive process, operating through a key addition transition state such as 58. 

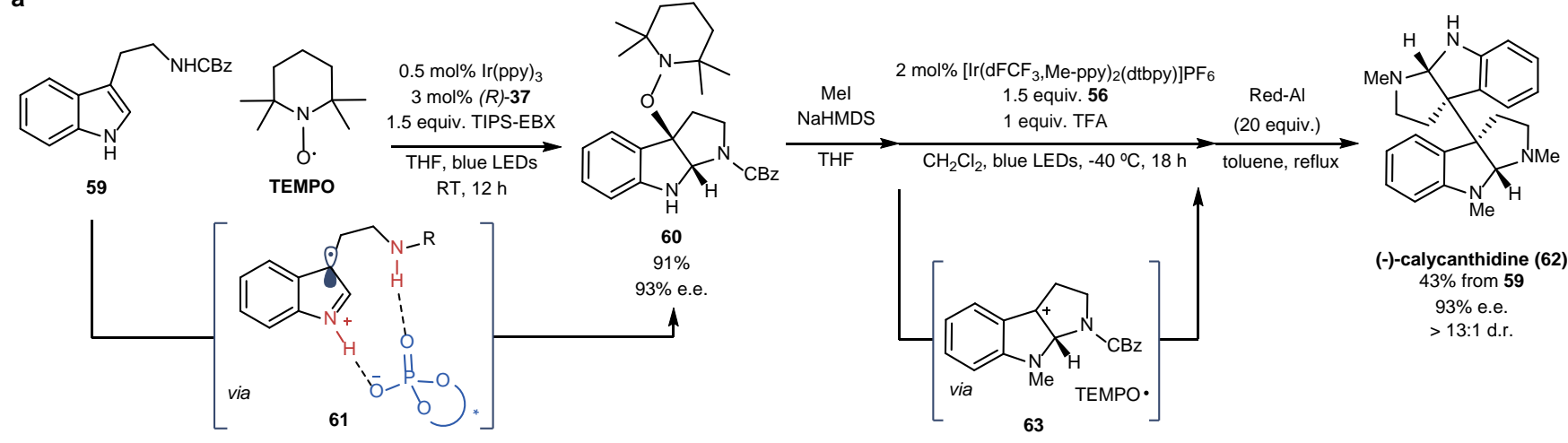
(-)-calycanthidine (62) 43\% from 59 93\% e.e. $>13: 1$ d.r.

b
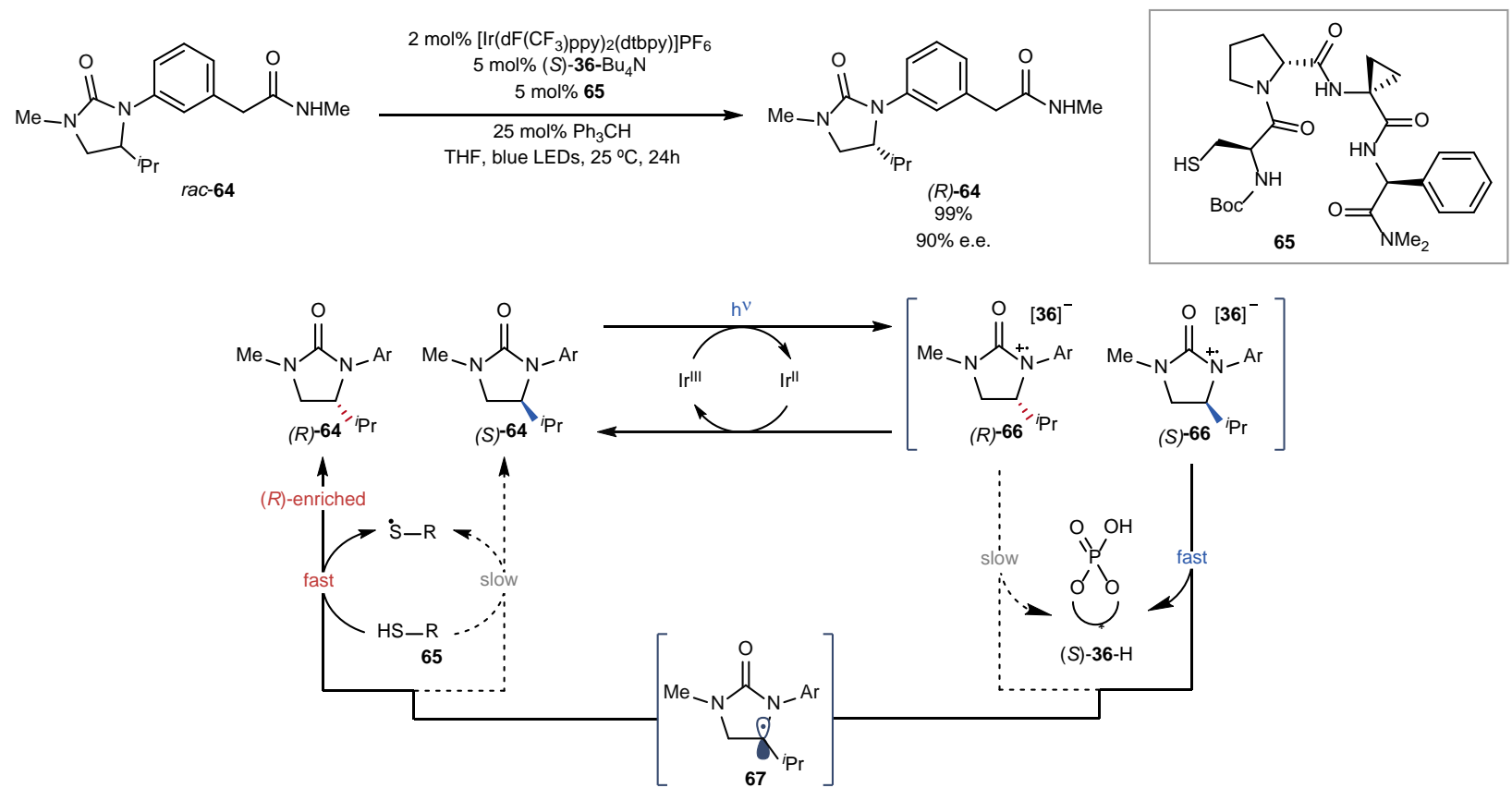

c
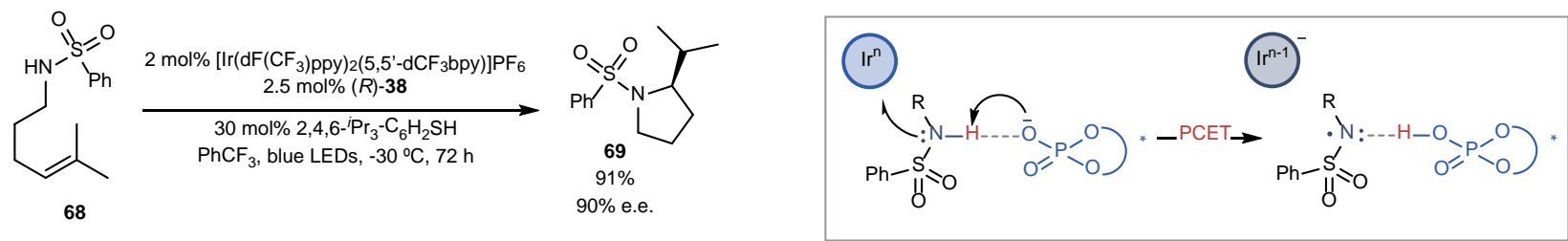

Figure 6 Recent advances in asymmetric radical processes using chiral phosphate catalysts. a, Synthesis of enantioenriched pyrroloindolines. Proton-coupled oxidation of 59 results in complex 61, in which the phosphate retains hydrogen bonding contacts with the distal radical cation. Radical-radical coupling with TEMPO followed by cyclisation of the pendant amide gives 60, which can be further derivatised in a stereospecific fashion via a key mesolytic cleavage and trapping with a nucleophile. b, Catalytic deracemisation of ureas by dual enantioselective catalysis. Rac-64 is oxidised to give $\mathbf{6 6}$, with enantiomer $(S)-\mathbf{6 6}$ selectively deprotonated by a chiral phosphate. The resultant neutral radical $\mathbf{6 7}$ can then undergo hydrogen atom transfer (HAT) from 65 to selectively form $(R)$-64. Both of these steps combine to enrich the enantiomeric ratio. $\mathbf{c}$, Intramolecular asymmetric hydroamination. After proton-coupled oxidation of sulfonamide $\mathbf{6 8}$, the chiral phosphate is proposed to remain $\mathrm{H}$-bonded to the neutral $\mathrm{N}$-centred radical, allowing asymmetric cyclisation to be achieved. 
<smiles>O=C1Nc2ccccc2C12Cc1ccccc1C2</smiles>

70<smiles>[O-][n+]1c(Cl)cccc1Cl</smiles>

71
$\stackrel{\substack{\mathrm{Ru}-72(1 \mathrm{~mol} \%) \\ \mathrm{CH}_{2} \mathrm{Cl}_{2}, 50^{\circ} \mathrm{C}, 20 \mathrm{~h}}}{\longrightarrow}$<smiles>CCCC</smiles>

2.2. equiv

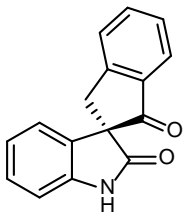

73

$70 \%$ yield, $80 \%$ e.e.

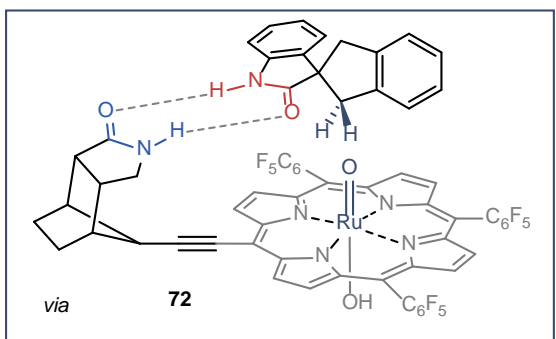<smiles>CC1c2ccc(C(c3ccccc3)c3ccccc3)cc2NC(=O)C1(C)C</smiles>
$\mathrm{CH}_{2} \mathrm{Cl}_{2}{ }^{(10 \mathrm{MIV}), \mathrm{O}^{\circ} \mathrm{C}, 10 \mathrm{IT}}$<smiles>CC1(C)C(=O)Nc2cc(Cc3ccccc3)ccc2[C@H]1O</smiles><smiles>C=C(CC(CO)CO)c1ccccc1</smiles>

82

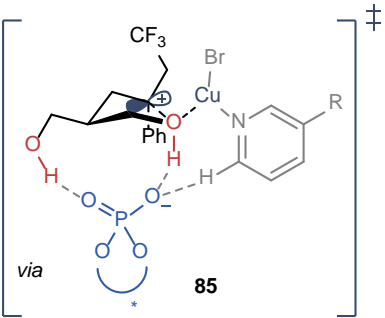

$10 \mathrm{~mol} \% \mathrm{Cu}_{2} \mathrm{O}$

$15 \mathrm{~mol} \%(R)-40$ $40 \mathrm{~mol} \% 84$ 1.5 equiv. 78 EtOOC $i \mathrm{Pr}$ $25^{\circ} \mathrm{C}, 96 \mathrm{~h}$

$$
\eta^{\ddagger}
$$

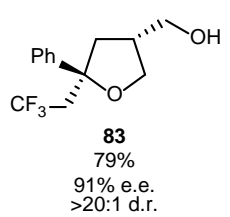

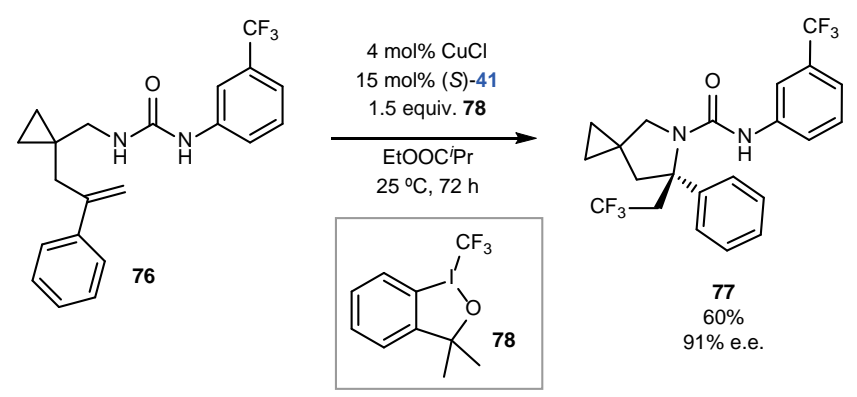

Figure 7 Non-covalent interactions in asymmetric radical-based organometallic reactions. a, A ruthenium porphyrin bearing a chiral lactam can enact desymmetrisation of spirocyclic compound $\mathbf{7 0}$ through hydrogen bonding in a radical rebound process. $\mathbf{b}$, Enantioselective benzylic oxidation of $\mathbf{7 4}$ by manganese-porphyrin $\mathbf{M n - 7 2}$ is also site-selective. The doubly benzylic methylene $\mathbf{C}-\mathrm{H}$ (highlighted in red) remains unaltered whilst double hydrogen bonding by the lactam elements in both substrate and catalyst directs the oxidation to occur exclusively at the singly benzylic C-4 methylene site (highlighted in blue). c, Asymmetric carboamination of styrenes by copper and a chiral phosphate ligand. Radical 79 may either undergo enantiodetermining single-electron addition to copper, guided by hydrogen bonds, to give $\mathbf{8 0}$ or oxidation by copper to form ion-paired complex $\mathbf{8 1}$ with subsequent asymmetric trapping of the resulting carbocation. $\mathbf{d}$, Desymmetrisation of diol $\mathbf{8 2}$ via carbocation $\mathbf{8 5}$ is orchestrated by multiple non-covalent contacts between the phosphate and copper complex during stepwise reductive elimination. 

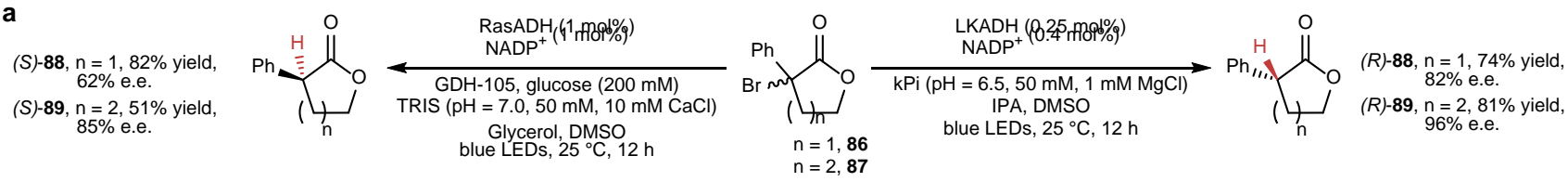

via
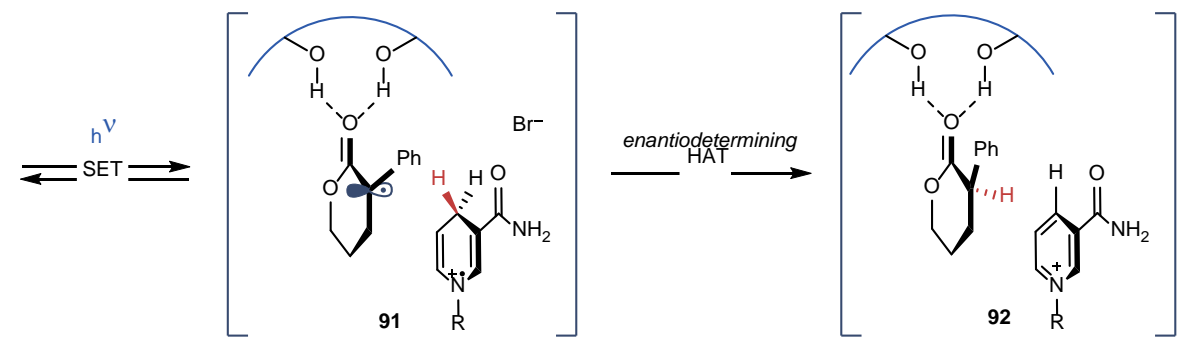<smiles>CC(=O)OC1(C)CCc2ccccc2C1=O</smiles>

93

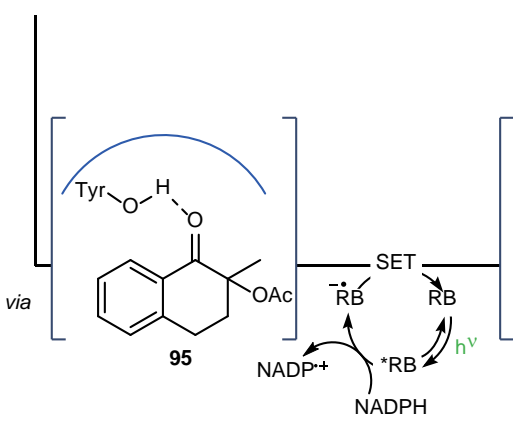

NADP Green LEDs, RT, $12 \mathrm{~h}$

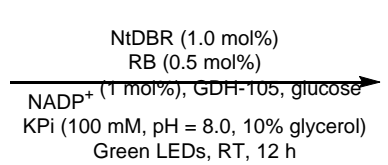

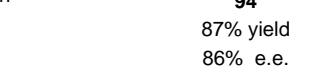

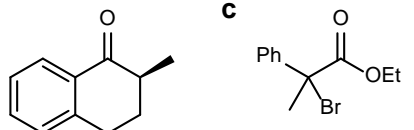

97

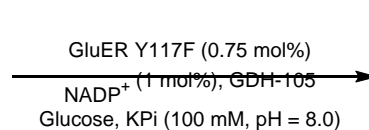

$10 \%$ IPA, $25{ }^{\circ} \mathrm{C}, 24 \mathrm{~h}$

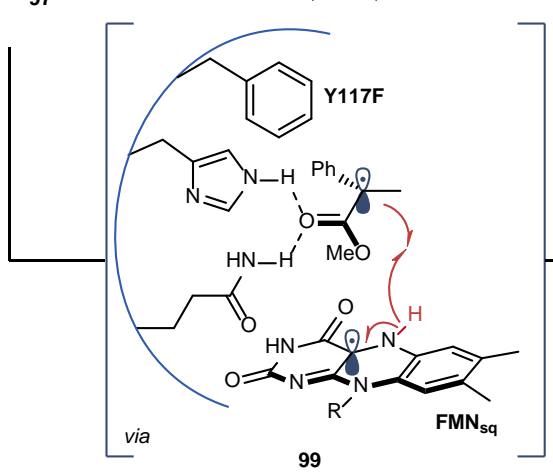<smiles>CCOC(=O)[C@H](C)c1ccccc1</smiles>
$87 \%$ yield $94 \%$ e.e.

d

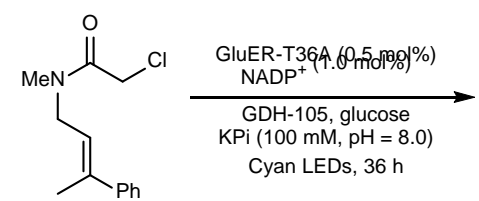

100

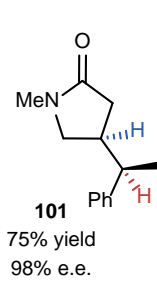

$92: 8$ d.r.

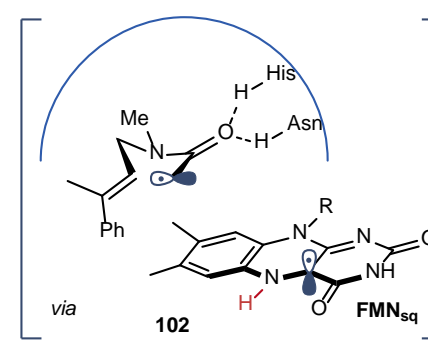

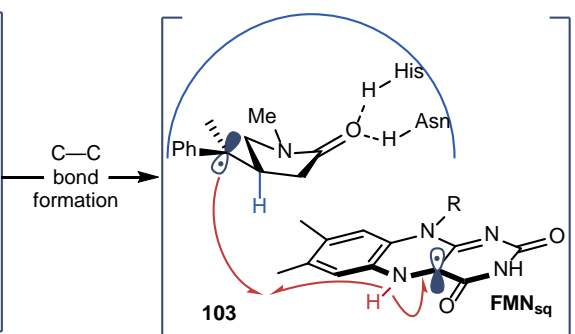

Figure 8 Examples demonstrating the use of biocatalysis to control enantioselectivity in non-natural radical reactions. a, Enantioselective dehalogenation of racemic lactones by photoexcitation of nicotinamide-dependent ketoreductases (KREDs). The LKADH KRED variant enabled access to the $R$ enantiomer of product while the RasADH KRED variant enabled access to the $S$ enantiomer of product. Positioning of the prochiral radical within the enzyme's active site by key hydrogen bonds from the substrate's carbonyl to amino acid residues dictates the facial selectivity of the enantiodetermining HAT event. b, Example of 'enzymatic redox activation' through the synergistic use of a nicotinamide-dependent double bond reductase (DBR), NtDBR, with Rose Bengal (RB) photocatalyst under visible light irradiation, for the enantioselective deacetoxylation of racemic tetralones. A hydrogen bond between the carbonyl of $\mathbf{9 3}$ and tyrosine residue of the enzyme active site attenuates the substrate's redox potential and exerts facial discrimination for enantiodetermining HAT. c, Enantioselective radical dehalogenation of $\alpha$-bromoesters using a flavin (FMN)dependent ene-reductase (ERED) catalyst, GluER Y117F. Bound to the enzyme's active site via hydrogen bonds, the prochiral $\alpha$-acyl radical selectively abstracts a hydrogen atom from flavin semiquinone $\left(\mathrm{FMN}_{\mathrm{sq}}\right) \mathbf{d}$, Enantioselective cyclisation of $\alpha$-chloroacetamides via photoexcitation of FMNdependent ERED, GluER-T36A. Hydrogen bonds between the substrate and histidine and asparagine residues of the enzyme render the cyclisation highly favourable. The enzyme controls the facial selectivity of the cyclisation (102) and subsequent exocyclic HAT from FMN sq $_{(103) .}$ 